\title{
The see-saw portal at future Higgs Factories
}

\author{
Daniele Barducci, ${ }^{a, b}$ Enrico Bertuzzo, ${ }^{c}$ Andrea Caputo, ${ }^{d, e}$ Pilar Hernandez ${ }^{f}$ \\ and Barbara Mele ${ }^{b}$ \\ ${ }^{a}$ Università degli Studi di Roma la Sapienza, \\ Piazzale Aldo Moro 5, 00185, Roma, Italy \\ ${ }^{b}$ INFN Section of Roma 1 , \\ Piazzale Aldo Moro 5, 00185, Roma, Italy \\ ${ }^{c}$ Instituto de Fisica, Universidade de Sao Paulo, \\ C.P. 66.318, 05315-970 Sao Paulo, Brazil \\ ${ }^{d}$ School of Physics and Astronomy, Tel-Aviv University, \\ Tel-Aviv 69978, Israel \\ ${ }^{e}$ Department of Particle Physics and Astrophysics, Weizmann Institute of Science, \\ Rehovot 7610001, Israel \\ ${ }^{f}$ Instituto de Física Corpuscular - CSIC/Universidad de Valencia, \\ Parc Cientufic de Paterna, Paterna, Spain \\ E-mail: daniele.barducci@roma1.infn.it, bertuzzo@if.usp.br, \\ andrea.caputo@uv.es, m.pilar.hernandez@uv.es, \\ barbara.mele@roma1.infn.it
}

ABSTRACT: We consider an extension of the Standard Model with two right-handed singlet fermions with mass at the electroweak scale that induce neutrino masses, plus a generic new physics sector at a higher scale $\Lambda$. We focus on the effective operators of lowest dimension $d=5$, which induce new production and decay modes for the singlet fermions. We assess the sensitivity of future Higgs Factories, such as FCC-ee, CLIC-380, ILC and CEPC, to the coefficients of these operators for various center of mass energies. We show that future lepton colliders can test the cut-off of the theory up to $\Lambda \simeq 500-1000 \mathrm{TeV}$, surpassing the reach of future indirect measurements of the Higgs and $Z$ boson widths. We also comment on the possibility of determining the underlying model flavor structure should a New Physics signal be observed, and on the impact of higher dimensional $d=6$ operators on the experimental signatures.

Keywords: Beyond Standard Model, Higgs Physics, Neutrino Physics

ArXiv EPRINT: 2011.04725 


\section{Contents}

1 Introduction 1

2 Theoretical framework 3

2.1 Neutrino mixing formalism 4

2.2 Heavy neutrinos decay modes 5

2.3 Final states from pair produced heavy neutrinos $\quad 7$

2.4 Heavy neutrinos lifetime 8

3 Future Higgs Factories $\quad 10$

$\begin{array}{ll}3.1 \text { Collider prototypes } & 10\end{array}$

$\begin{array}{lll}3.2 & \text { Simulation framework } & 10\end{array}$

4 The $\mathcal{O}_{N H}$ operator and the Higgs-strahlung channel $\quad 11$

$\begin{array}{lll}4.1 & \text { Prompt decay } & 12\end{array}$

4.1.1 Projected sensitivities: $e$ and $\mu$ mixing 12

4.1.2 Projected sensitivities: $\tau$ mixing $\quad 15$

$\begin{array}{ll}\text { 4.1.3 Determination of the flavor structure } & 16\end{array}$

$\begin{array}{ll}4.2 \text { Displaced decay } & 17\end{array}$

$\begin{array}{lll}4.3 & \text { Detector stable } & 19\end{array}$

5 The $\mathcal{O}_{N B}$ operator and the s-channel $Z$ production 20

5.1 Prompt decay 22

5.2 Displaced decay 24

$\begin{array}{lll}5.3 & \text { Detector stable } & 25\end{array}$

6 The impact of $d=6$ operators $\quad 26$

$\begin{array}{lll}7 & \text { Conclusions } & 27\end{array}$

\section{Introduction}

The discovery of neutrino masses and oscillations is one of the most striking evidences for the need of new physics (NP) beyond the Standard Model (SM). Arguably, the simplest extension of the SM consists in extending its field content with the right-handed $(\mathrm{RH})$ counterparts of the left-handed SM neutrinos, $N$. At the renormalizable level this allows for Yukawa type interactions between the new states and the SM leptons, providing a Dirac mass term for the neutrinos. However, being electroweak (EW) and color singlets, the new states can also have a Majorana mass terms, $M_{N}$. As it is well known, this allows 
to explain the lightness of the observed neutrino masses through a large hierarchy between the EW scale and the mass scale of the RH neutrinos

$$
m_{\nu} \propto y^{2} \frac{v^{2}}{M_{N}},
$$

where $y$ is the strength of the Yukawa interaction and $v$ the Higgs vacuum expectation value (VEV). The relation of eq. (1.1) defines the see-saw mechanism [1-4]. For a natural choice of the Yukawa interactions, $y=\mathcal{O}(1)$, the lightness of neutrino masses requires $\mathrm{RH}$ neutrinos at around the Grand Unification scale. However see-saw models with EW-scale $\mathrm{RH}$ neutrinos have recently received increasing attention. On the one side they offer a compelling alternative for the generation of the matter-antimatter asymmetry via neutrino oscillations [5, 6], while on the other side they can be searched for at colliders and in beam-dump experiments [7-24].

At the renormalizable level these extra states can only be produced or decay via their mixing with the active neutrinos. This mixing, that controls their charged- and neutralcurrent interactions, is given by

$$
\theta \propto \frac{y v}{M_{N}} .
$$

For EW scale RH neutrinos the naive see-saw scaling of eq. (1.1) requires a tiny value for the Yukawa coupling connecting the SM and the beyond the SM (BSM) sectors. This implies a tiny mixing of the $\mathrm{RH}$ neutrino, resulting in a small production cross-section and a small decay width. The latter can give rise to striking signatures, such as displaced decays.

Interestingly, both the properties of production via mixing and displaced decays of the $\mathrm{RH}$ neutrinos can be challenged. The naive see-saw scaling relation can be broken when more than one $\mathrm{RH}$ state is present by specific Yukawa and Majorana mass textures that ensure an approximate lepton number symmetry [25, 26]. The mixing can be much larger than the one implied by the see-saw relation, thus modifying the lifetime of the BSM states. $\mathrm{RH}$ neutrinos can then feature a prompt, displaced or detector stable behaviour.

These predictions can also be altered by the presence of additional NP states at a scale $\Lambda \gg v, M$. At low energy their effects can be described in the language of effective field theories (EFT) by a tower of higher dimensional operators $\mathcal{O}^{d} \Lambda^{4-d}$ with $d>4$, built out from the SM and RH neutrinos fields: the $\nu$ SMEFT. At the lowest dimension, $d=5$, two new operators intervene to induce new RH neutrinos production modes: an operator triggering a new Higgs decay channel into a pair of RH neutrinos and a dipole operator connecting the $\mathrm{RH}$ neutrino tensor current with the hypercharge gauge boson $[27,28]$. At $d=6$ many more operators are present $[9,29,30]$, which can induce new production as well as new decay channels.

Various theoretical studies have investigated the signatures at the Large Hadron Collider (LHC) of a subset of these higher dimensional operators, see e.g. [9, 21, 27, 28, 31-33]. The search for EW scale RH neutrinos is however one the primary goal of future $e^{+} e^{-}$colliders, thanks to the clean detector environment and the tipically lower SM backgrounds with respect to an hadronic machine, which can help to overcome the generally small production cross-sections of SM singlet states. Various future prototypes has been designed for 
the post LHC era: both circular colliders, as the Future Circular Collider [34-37] (FCC-ee) and the Compact electron-positron collider [38, 39] (CEPC), and linear ones, such as the International Linear Collider [40-42] (ILC) and the Compact Linear Collider [43, 44] (CLIC). It is then the purpose of this paper to investigate the phenomenology of the $\nu \mathrm{SMEFT}$ at these future machines and study their sensitivity on the $d>4$ operators inducing new $\mathrm{RH}$ neutrinos production and decay modes in all the possible regimes of the $N$ decay lifetimes, for $\mathrm{RH}$ masses in the range $1-60 \mathrm{GeV}$.

The paper is organized as follows. In section 2 we introduce our notation and describe the active-sterile mixing formalism while in section 3 we discuss the properties of the various $e^{+} e^{-}$colliders under analysis. In section 4 and in section 5 we describe the analysis details and show the projected reach of the collider prototypes on the $d=5$ operators involving $\mathrm{RH}$ neutrino fields, while in section 6 we discuss the possible impact of $d=6$ operators inducing extra $N$ production and decay modes. We then conclude in section 7 .

\section{Theoretical framework}

We work in the framework of the $\nu$ SMEFT, which is described by the following Lagrangian

$$
\mathcal{L}=\mathcal{L}_{\mathrm{SM}}+\bar{N} \not \partial N-\bar{L}_{L} Y_{\nu} \tilde{H} N-\frac{1}{2} M_{N} \bar{N}^{c} N+\sum_{n>4} \frac{\mathcal{O}^{n}}{\Lambda^{n-4}}+\text { h.c. }
$$

where $N$ is vector describing $\mathcal{N}$ flavors of gauge singlet RH neutrino fields with $N^{c}=C \bar{N}^{T}$ and $C=i \gamma^{2} \gamma^{0}, L$ is the SM lepton doublet, $Y_{\nu}$ is the $3 \times \mathcal{N}$ Yukawa matrix of the neutrino sector with $\tilde{H}=i \sigma^{2} H^{*}, M_{N}$ is a $\mathcal{N} \times \mathcal{N}$ Majorana mass matrix for the RH neutrino fields and $\mathcal{O}^{n}$ the Lorentz and gauge invariant operators built out from the SM and the $\mathrm{RH}$ neutrino fields. The $\nu$ SMEFT has been constructed up to $d=7$ in $[9,27-30]$. At dimension five only three operators exist

$$
\begin{aligned}
\mathcal{O}_{W} & =\alpha_{W}\left(\bar{L}^{c} \tilde{H}^{*}\right)\left(\tilde{H}^{\dagger} L\right), \\
\mathcal{O}_{N H} & =\alpha_{N H}\left(\bar{N}^{c} N\right)\left(H^{\dagger} H\right), \\
\mathcal{O}_{N B} & =\alpha_{N B} \bar{N}^{c} \sigma^{\mu \nu} N B_{\mu \nu},
\end{aligned}
$$

where $\alpha_{W}$ and $\alpha_{N H}$ are symmetric $3 \times 3$ and $\mathcal{N} \times \mathcal{N}$ matrices in flavor space respectively, $\alpha_{N B}$ is an antisymmetric $\mathcal{N} \times \mathcal{N}$ matrix in flavor space, $\sigma^{\mu \nu}=i / 2\left[\gamma^{\mu}, \gamma^{\nu}\right]$ and $B_{\mu \nu}$ is the SM hypercharge field strength tensor. The first operator is the well known Weinberg operator [45] responsible for generating a Majorana mass for the SM neutrinos. The operator $\mathcal{O}_{N H}$ induces new interactions between the Higgs field and the $\mathrm{RH}$ neutrinos and adds an extra contribution to the $\mathrm{RH}$ neutrinos Majorana mass matrix, while $\mathcal{O}_{N B}$ is a dipole type operator connecting the $\mathrm{RH}$ neutrino tensor current to the hypercharge gauge boson. The operator coefficients $\alpha_{W}$ and $\alpha_{N H}$ can be $\mathcal{O}(1)$, while $\alpha_{N B}$ is necessarily $\mathcal{O}\left(1 / 16 \pi^{2}\right)$ since it can be only generated at loop level in a weakly coupled ultraviolet completion of the effective Lagrangian of eq. (2.1). 


\subsection{Neutrino mixing formalism}

Without loss of generality it is possible to go from eq. (2.1) to a basis where the matrix $M_{N}$ and the charged lepton mass matrix are diagonal with non negative entries. Working at $d=5$, the operator $\mathcal{O}_{N H}$ contributes to the neutrino mass matrix. By defining $n=\left(\nu_{L}, N^{c}\right)$ and using $\langle H\rangle=174 \mathrm{GeV}$, the mass Lagrangian in the neutrino sector can be written as

$$
\mathcal{L}_{\text {mass }}=-\frac{1}{2} \bar{n}^{c} \mathcal{M} n+\text { h.c. }=-\frac{1}{2} \bar{n}^{c}\left(\begin{array}{cc}
-2 \alpha_{W} \frac{v^{2}}{\Lambda} & Y_{\nu} v \\
Y_{\nu}^{T} v & M_{N}-2 \alpha_{N H} \frac{v^{2}}{\Lambda}
\end{array}\right) n+\text { h.c. },
$$

where the $\nu_{L}-\nu_{L}$ block receives a contribution only from the $d=5$ Weinberg operator while the $N-N$ one has both $d=4$ and $d=5$ contributions. This mass matrix can be perturbatively diagonalized in the regime in which the entries of the $\nu_{L}-N$ block are smaller than the ones in the $N-N$ one. For our purposes we assume that the see-saw contribution to the active neutrino masses dominates over the other ones. Under this approximation we obtain

$$
m_{\nu} \simeq v^{2} Y_{\nu} \frac{1}{M_{N}} Y_{\nu}^{T}=U^{*} m_{\nu}^{(d)} U^{\dagger}
$$

where $m_{\nu}^{(d)}$ is diagonal with non negative entries and $U$ is the Pontecorvo-Maki-NakagawaSakata (PMNS) matrix [46, 47]. From eq. (2.4) one can obtain

$$
Y_{\nu} \simeq \frac{1}{v} U^{*} \sqrt{\mu} \sqrt{M_{N}}
$$

where the $3 \times \mathcal{N}$ matrix $\sqrt{\mu}$ satisfies $\sqrt{\mu} \sqrt{\mu}^{T}=m_{\nu}^{(d)}$ and $\sqrt{\mu}$ and $\sqrt{M_{N}}$ indicate, respectively, $\mu^{1 / 2}$ and $M_{N}^{1 / 2}$. The usefulness of this parametrization is that it allows to write in a compact way the expressions for the various matrices involved. We now restrict our analysis to the case of two $\mathrm{RH}$ neutrinos, thus fixing $\mathcal{N}=2$. Without loss of generality the matrix $\sqrt{\mu}$ can be written using the so-called Casas-Ibarra parametrization [48] as

$$
\sqrt{\mu}=\sqrt{m} \mathcal{R},
$$

where $\sqrt{m}$ is a $3 \times 2$ matrix containing the physical neutrino masses $m_{i}$, while $\mathcal{R}$ is a complex orthogonal $2 \times 2$ matrix, $\mathcal{R}^{T} \mathcal{R}=\mathbb{1}$. With two RH neutrinos one has $m_{\nu_{1}}=0$ and $m_{\nu_{3}}>m_{\nu_{2}}$ in the normal hierarchy (NH) case, while $m_{\nu_{3}}=0$ and $m_{\nu_{2}}>m_{\nu_{1}}$ in the inverted hierarchy (IH) one. ${ }^{1}$ More in detail, for NH and inverted IH we have

$$
\sqrt{m_{N H}}=\left(\begin{array}{cc}
0 & 0 \\
0 & \sqrt{m_{2}} \\
\sqrt{m_{3}} & 0
\end{array}\right), \quad \sqrt{m_{I H}}=\left(\begin{array}{cc}
0 & \sqrt{m_{1}} \\
\sqrt{m_{2}} & 0 \\
0 & 0
\end{array}\right),
$$

while we parametrize the orthogonal matrix $\mathcal{R}$ in terms of the complex angle $z=\beta+i \gamma$ as

$$
\mathcal{R}=\left(\begin{array}{cc}
\cos z & \pm \sin z \\
-\sin z & \pm \cos z
\end{array}\right)
$$

\footnotetext{
${ }^{1}$ For the NH we take $m_{\nu_{2}}=8.6 \times 10^{-3} \mathrm{eV}$ and $m_{\nu_{3}}=5.1 \times 10^{-2} \mathrm{eV}$ while for the IH we take $m_{\nu_{1}}=$ $4.9 \times 10^{-2} \mathrm{eV}$ and $m_{\nu_{2}}=5.0 \times 10^{-2} \mathrm{eV}$.
} 
For both hierarchies we can thus write

$$
Y_{\nu} \simeq \frac{1}{v} U^{*} \sqrt{m} \mathcal{R} \sqrt{M_{N}},
$$

where $m=m_{\mathrm{NH}}$ or $m_{\mathrm{IH}}$, and obtain a compact expression for the active-sterile mixing angle

$$
\theta_{\nu N} \simeq-U^{*} \sqrt{m} \mathcal{R} \frac{1}{\sqrt{M_{N}}} .
$$

It's crucial that the angle $z$ can be taken in general as a complex number. In fact, in the limit in which $z$ is a real number, by taking $U$ and $\mathcal{R}$ with entries of order unity and by assuming an equal value for the diagonal entries of the Majorana mass term for the two RH neutrino $m_{N_{1}}=m_{N_{2}}=m_{N}$, one obtains ${ }^{2}$

$$
Y_{\nu} \sim \frac{\sqrt{m_{N}} m_{\nu}}{v} \sim 4 \times 10^{-8}\left(\frac{m_{N}}{1 \mathrm{GeV}}\right)^{1 / 2} .
$$

This "naive see-saw scaling" relation is drastically modified by the imaginary part of $z$, that gives an exponential enhancement. In the limit $\gamma \gg 1$

$$
\mathcal{R} \simeq \frac{e^{\gamma-i \beta}}{2}\left(\begin{array}{cc}
1 & \pm i \\
-i & \pm 1
\end{array}\right)
$$

and the relation of eq. (2.11) is modified to

$$
Y_{\nu} \sim 2 \times 10^{-8} e^{\gamma-i \beta}\left(\frac{m_{N}}{1 \mathrm{GeV}}\right)^{1 / 2} .
$$

The same enhancement is inherited by the active-sterile mixing, that now reads

$$
\theta_{\alpha i} \equiv\left(\theta_{\nu N}\right)_{\alpha i} \sim 7.2 \times 10^{-6} e^{\gamma-i \beta}\left(\frac{1 \mathrm{GeV}}{m_{N}}\right)^{1 / 2} .
$$

In the previous expression we have $\alpha=e, \mu, \tau$ and $i=1,2$. As anticipated in the Introduction and as we will see more in detail below, this deviation from the naive see-saw scaling has a crucial impact on the RH neutrinos phenomenology, especially for what concerns their decay width and consequently their lifetime, with huge implications for search strategies at future colliders.

\subsection{Heavy neutrinos decay modes}

In the mass range of our interest and at the renormalizable level the $\mathrm{RH}$ neutrinos can only decay through charged- and neutral-currents via an off-shell $W$ or $Z$ boson. ${ }^{3}$ The RH neutrino decay mode is thus completely fixed once the $W$ and $Z$ decay channels are specified. The various final states from $N$ decay are reported in table 1 where $\alpha, \beta, i$ and

\footnotetext{
${ }^{2}$ We have assumed NH and fixed $m_{\nu}=m_{\nu_{3}}$. The expression holds also for the IH case modulo order one factors.

${ }^{3} \mathrm{~A}$ decay into an off-shell Higgs boson is generally suppressed by the smallness of the SM Yukawa couplings.
} 


\begin{tabular}{|c|c|c|}
\hline Final state & Channel & Mediator \\
\hline$\ell^{\prime} q \bar{q}$ & $\ell_{\alpha}^{\prime} q_{i} \bar{q}_{j}$ & $W$ \\
\hline$\nu q \bar{q}$ & $\nu_{\alpha} q_{i} \bar{q}_{j}$ & $Z$ \\
\hline & $\ell_{\alpha}^{\prime} \ell_{\beta}^{\prime} \nu_{\beta}, \alpha \neq \beta$ & $W$ \\
$\nu \ell^{\prime} \ell^{\prime}$ & $\nu_{\alpha} \ell_{\beta}^{\prime} \ell_{\beta}^{\prime}, \alpha \neq \beta$ & $Z$ \\
& $\nu_{\alpha} \ell_{\beta}^{\prime} \ell_{\beta}^{\prime}, \alpha=\beta$ & $W$ and $Z$ \\
\hline$\nu \nu \nu$ & $\nu_{\alpha} \nu_{\beta} \nu_{\beta}$ & $Z$ \\
\hline
\end{tabular}

Table 1. Possible decay channels for the RH neutrino $N$. Here $\alpha, \beta, i$ and $j$ are flavor indices and we do not specify the charge of the charged lepton $\ell^{\prime}=e, \mu, \tau$ nor the nature of the (anti)neutrino.

$j$ are flavor indices and, for simplicity, we do not specify neither the charge of the charged lepton $\ell^{\prime}=e, \mu, \tau$ nor the nature of the (anti)neutrino. In this table we have grouped together the three decay modes giving rise to the $\nu \ell^{\prime} \ell^{\prime}$ final state since, given that both the $W$ and $Z$ boson will be non resonant for the RH neutrinos mass range of our interest, these processes will not be distinguishable. Moreover, the $\nu_{\alpha} \ell_{\beta}^{\prime} \ell_{\beta}^{\prime}$ process with $\alpha=\beta$ receives contributions from both neutral- and charged-current interactions, which interfere among themselves.

For computing the partial widths into the final state of table 1 we use the results of [49]. For $m_{N} \gg \Lambda_{\mathrm{QCD}}$, the decay rates involving quark pairs are physical quantities, otherwise decays into hadrons should instead be considered. Following again [49] we have implemented three-loop QCD corrections through which the full hadronic width can be computed from the decay width into free quarks. Altogether the effect on the total width is found to be around $30 \%$ for $m_{N} \simeq 1 \mathrm{GeV}$, decreasing down to $10 \%$ for $m_{N} \simeq 5 \mathrm{GeV}$. By fixing the phases of the PMNS matrix $\delta=\phi_{1}=0,{ }^{4}$ and also $\gamma=\beta=0$ we obtain the branching ratios (BRs) shown in the left panel of figure 1 for the case of the lightest $\mathrm{RH}$ neutrino $N_{1}$, while similar rates are obtained for $N_{2}$.

The lepton flavor composition of the final states depends on the active-sterile mixing matrix, which in turn depends on $i$ ) the hierarchy and the squared mass differences of the active neutrinos, ii) the phases of the PMNS matrix $\delta$ and $\phi_{1}$, and iii) the $\beta$ and $\gamma$ parameters entering in the Casas-Ibarra parametrization of eq. (2.8). Analytical approximations for the various mixings can be derived when $\gamma \gtrsim 1$, see e.g. [50]. In this regime the Casas-Ibarra parameter $\beta$ has a little impact on the normalized squared mixing

$$
r_{\alpha i}^{2}=\frac{\left|\theta_{\alpha i}\right|^{2}}{\bar{U}_{i}^{2}}, \quad \bar{U}_{i}^{2}=\sum_{\alpha=e, \mu, \tau}\left|\theta_{\alpha i}\right|^{2}, \quad \alpha=e, \mu, \tau, \quad i=1,2
$$

which is mainly determined by the PMSN phases $\delta$ and $\phi_{1}$. By varying them between $[0,2 \pi]$ we obtain for $r_{\alpha 1}^{2}$ the allowed ranges shown in the right panel of figure 1 . The red and blue regions correspond to $\mathrm{NH}$ and $\mathrm{IH}$, respectively, and $r_{\tau 1}^{2}=1-r_{e 1}^{2}-r_{\mu 1}^{2}$, see also [21]. We also show the benchmark points that will be used in the following analysis.

\footnotetext{
${ }^{4}$ We remind the reader that with $\mathcal{N}=2 \mathrm{RH}$ states only two phases are present in the PMNS matrix. We denote by $\delta$ the so-called Dirac phase, and by $\phi_{1}$ the so-called Majorana phase.
} 

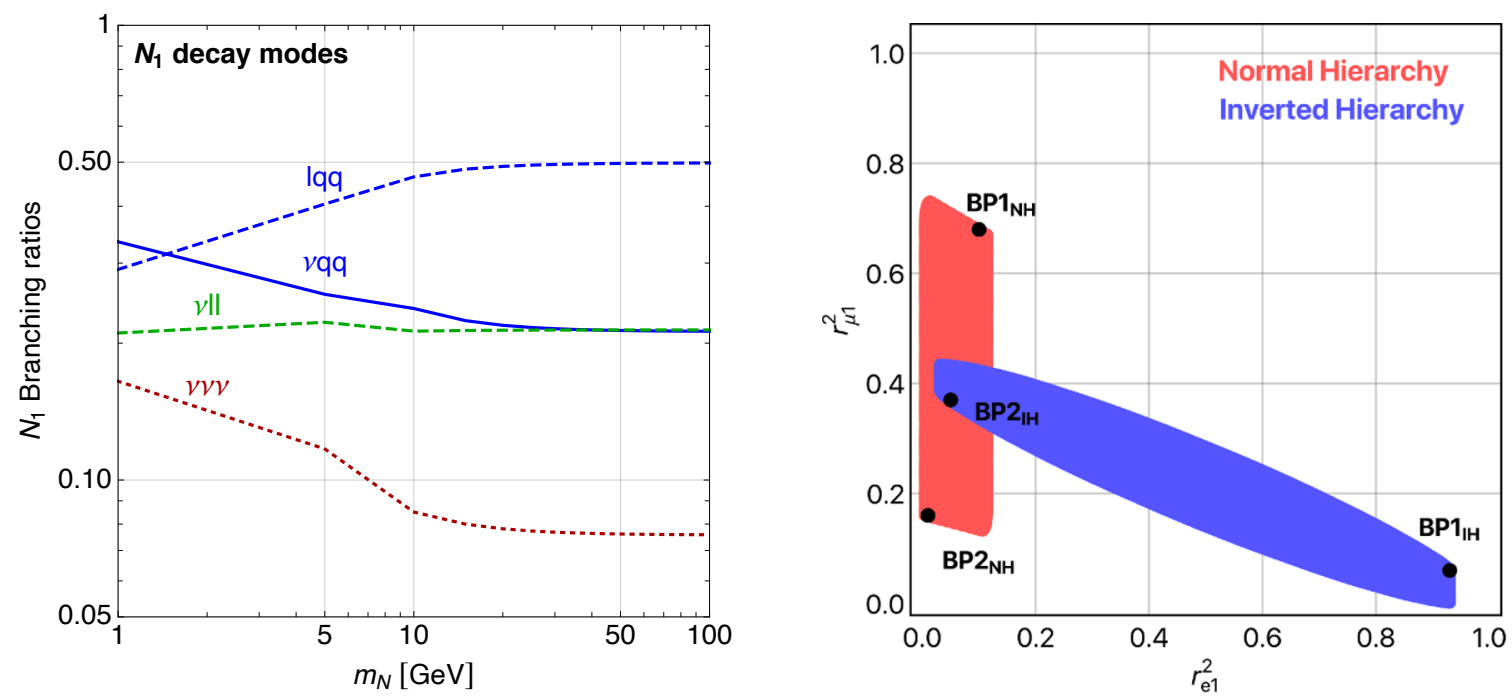

Figure 1. Left: decay modes of the lightest RH neutrino $N_{1}$. We have fixed $\delta=\phi_{1}=\beta=\gamma=0$. Right: allowed ranges in the $r_{e 1}^{2}-r_{\mu 1}^{2}$ plane for $\gamma \gtrsim 1$, obtained by varying the PMNS phases $\delta$ and $\phi$ in the range $[0,2 \pi]$ with $r_{\tau 1}=1-r_{e 1}^{2}-r_{\mu 1}^{2}$. Also shown are the benchmark points used in the analysis.

\subsection{Final states from pair produced heavy neutrinos}

Both the operators $\mathcal{O}_{N H}$ and $\mathcal{O}_{N B}$ will mediate the production of a pair of $\mathrm{RH}$ neutrinos, which in turn will decay producing a six-body final state. These final states can be categorized into fully-leptonic, fully-hadronic, semi-leptonic and invisible channels and are reported in table 2 . Here we differentiate between $\ell=e, \mu$ and $\tau$, since the latter particle decays within the detector thus producing a different and more complex final state. With an abuse of notation we nevertheless use the nomenclature fully-leptonic and semi-leptonic also for the final states involving $\tau$ leptons. Particularly interesting are the final states that can present a pair of same-sign (SS) leptons $\ell=e, \mu$, a signature which generally has a low SM background. Final states with $\geq 3 \ell$ will clearly have a SS pair. However, also the final state with exactly $2 \ell$ can produce a SS signature, due to the Majorana nature of the $\mathrm{RH}$ neutrinos. The branching ratios of $N_{1}$ and $N_{2}$ into the various final states of table 2 will depend upon the choice of the normalized squared mixings $r_{\alpha i}^{2}$ which, as explained above, depend on the PMNS parameter $\delta$ and $\phi_{1}$ and can span the ranges illustrated on the right panel of figure 1 .

For what concerns the detection and reconstruction of the final state, $e$ and $\mu$ can be considered in first approximation on the same footage, while one needs to distinguish them with respect to $\tau$ leptons. We then choose to perform our analysis and illustrate our results for some representative points in the allowed range for the $r_{\alpha i}^{2}$ shown in figure 1 for both the NH and IH cases. In particular for each mass hierarchy we choose two points, one with a large and one with a small mixing with the third generation leptons. After having fixed $r_{\tau i}^{2}$ we choose to maximize the mixing with the electron, which does not affect the reconstruction of the final state, in the approximation of similar $e$ and $\mu$ detection efficiencies, 


\begin{tabular}{|c|c|c|}
\hline & Channel & SS \\
\hline \multirow{5}{*}{ Fully-leptonic } & $4 \ell \mathbb{E}_{T}$ & $\checkmark$ \\
& $2 \ell \mathbb{E}_{T}$ & \\
\hline & $3 \ell 2 q \mathbb{E}_{T}$ & $\checkmark$ \\
& $2 \ell 4 q$ & $\checkmark$ \\
Semi-leptonic & $2 \ell 2 q \mathbb{E}_{T}$ & \\
& $\ell 4 q \mathbb{E}_{T}$ & \\
& $\ell 2 q \mathbb{E}_{T}$ & \\
\hline \multirow{2}{*}{ Fully-hadronic } & $4 q \mathbb{E}_{T}$ & \\
& $2 q \mathbb{E}_{T}$ & \\
\hline Invisible & $\mathbb{E}_{T}$ & \\
\hline
\end{tabular}

\begin{tabular}{|c|c|c|}
\hline & Channel & SS \\
\hline & $3 \ell \tau \mathbb{E}_{T}$ & $\checkmark$ \\
& $2 \ell 2 \tau \mathbb{E}_{T}$ & \\
$\ell \tau$ & $\mathbb{E}_{T}$ & \\
& $\ell \tau \mathbb{E}_{T}$ & \\
$4 \tau \mathbb{E}_{T}$ & \\
& $2 \tau \mathbb{E}_{T}$ & \\
\hline \multirow{5}{*}{ Semi-leptonic } & $2 \ell \tau 2 q \mathbb{E}_{T}$ & \\
& $\ell 2 \tau 2 q \mathbb{E}_{T}$ & \\
& $\ell \tau 4 q$ & \\
& $\ell \tau 2 q \mathbb{E}_{T}$ & \\
\hline
\end{tabular}

\begin{tabular}{|c|c|c|}
\hline & Channel & SS \\
\hline Semi-leptonic & $\begin{array}{c}3 \tau 2 q \mathbb{E}_{T} \\
2 \tau 4 q \\
2 \tau 2 q \mathbb{E}_{T} \\
\tau 2 q \mathbb{E}_{T} \\
\tau 4 q \mathbb{E}_{T}\end{array}$ & \\
\hline
\end{tabular}

Table 2. Possible final states from the decay of pair produced RH neutrinos. The checkmarks correspond to channels that can produce a SS leptons signal. The leftmost and rightmost tables contains final states without $\tau$ leptons and light leptons, $\ell$, respectively.

but does intervene in the production of $\mathrm{RH}$ neutrinos via mixing, $e^{+} e^{-} \rightarrow \nu N$. The reason why we choose to maximize $r_{e \alpha}^{2}$ is because, conservatively, we want to analyze a configuration with the largest possible production cross-section via mixing, to see whether the additional production modes arising from the $\mathcal{O}_{N H}$ and $\mathcal{O}_{N B}$ operators can still dominate over it. More concretely, we choose the following two benchmark points for the NH case

$$
\begin{array}{ll}
\mathbf{B P 1} 1_{\mathrm{NH}}: & r_{e 4}^{2}: r_{\mu 4}^{2}: r_{\tau 4}^{2}=0.10: 0.68: 0.22 \\
\mathbf{B P 2} \mathbf{N H}_{\mathbf{N H}}: & r_{e 4}^{2}: r_{\mu 4}^{2}: r_{\tau 4}^{2}=0.01: 0.16: 0.83
\end{array}
$$

which, in the $\gamma>1$ and degenerate mass limit, can be realized simultaneously for both $N_{1}$ and $N_{2}$, which therefore have similar BRs. The corresponding $N N$ decay rates are reported in table 3. Similarly, we choose the following benchmark points for the IH case

$$
\begin{array}{ll}
\mathbf{B P 1}_{\mathbf{I H}}: & r_{e 4}^{2}: r_{\mu 4}^{2}: r_{\tau 4}^{2}=0.93: 0.06: 0.01 \\
\mathbf{B P 2}_{\mathbf{I H}}: & r_{e 4}^{2}: r_{\mu 4}^{2}: r_{\tau 4}^{2}=0.05: 0.37: 0.58,
\end{array}
$$

whose BRs are reported in table 4.

\subsection{Heavy neutrinos lifetime}

A crucial quantity affecting the phenomenology and thus the search strategies for $\mathrm{RH}$ neutrinos is their lifetime $\tau_{N}=1 / \Gamma_{N}$. We focus for simplicity on the case of (almost) degenerate $\mathrm{RH}$ neutrinos and we start by considering only the decay modes of section 2.2 that are induced at the renormalizable level by the active-sterile mixing. ${ }^{5}$ In particular, as we will show in section 4.1, one can have RH neutrinos that decay promptly, displaced or are stable on detector length scales.

\footnotetext{
${ }^{5}$ These decay modes turns out to be the dominant ones also in the presence of $d=6$ operators when the minimal flavor violation paradigm is imposed [51].
} 


\begin{tabular}{|c|c|c|c|c|c|}
\hline BR & Channel & SS & BR & Channel & SS \\
\hline 0.16 & $2 \ell 4 q$ & $\checkmark$ & 0.01 & $3 \ell \tau \mathbb{E}_{T}$ & \\
0.09 & $\ell 4 q \mathbb{E}_{T}$ & & 0.01 & $2 \ell 2 \tau \mathbb{E}_{T}$ & \\
0.05 & $4 q \mathbb{E}_{T}$ & & 0.01 & $2 \ell \mathbb{E}_{T}$ & \\
0.05 & $2 \ell \tau 2 q \mathbb{E}_{T}$ & & 0.01 & $2 \tau 4 q$ & \\
0.04 & $3 \ell 2 q \mathbb{E}_{T}$ & $\checkmark$ & 0.01 & $\ell \tau \mathbb{E}_{T}$ & \\
0.03 & $\ell 4 q \tau$ & & 0.01 & $\tau 2 q \mathbb{E}_{T}$ & \\
0.03 & $\ell 2 q \mathbb{E}_{T}$ & & 0.01 & $\mathbb{E}_{T}$ & \\
0.02 & $2 \ell 2 q \mathbb{E}_{T}$ & & 0. & $2 \tau 2 q \mathbb{E}_{T}$ & \\
0.02 & $\ell \tau 2 q \mathbb{E}_{T}$ & & 0. & $\ell 3 \tau \mathbb{E}_{T}$ & \\
0.02 & $\tau 4 q \mathbb{E}_{T}$ & & 0. & $3 \tau 2 q \mathbb{E}_{T}$ & \\
0.02 & $2 q \mathbb{E}_{T}$ & & 0. & $2 \tau \mathbb{E}_{T}$ & \\
0.01 & $\ell 2 \tau 2 q \mathbb{E}_{T}$ & & 0. & $4 \tau \mathbb{E}_{T}$ & \\
0.01 & $4 \ell \mathbb{E}_{T}$ & $\checkmark$ & & & \\
\hline
\end{tabular}

\begin{tabular}{|c|c|c|c|c|c|}
\hline BR & Channel & SS & BR & Channel & SS \\
\hline 0.13 & $2 \tau 4 q$ & & 0.01 & $2 \tau 2 q \mathbb{E}_{T}$ & $\checkmark$ \\
0.09 & $\tau 4 q \mathbb{E}_{T}$ & & 0.01 & $2 \ell 4 q$ & \\
0.06 & $4 q \mathbb{E}_{T}$ & & 0.01 & $\ell 2 q \mathbb{E}_{T}$ & \\
0.06 & $\ell 2 \tau 2 q \mathbb{E}_{T}$ & & 0.01 & $\mathbb{E}_{T}$ & \\
0.04 & $\ell \tau 2 q \mathbb{E}_{T}$ & & 0.01 & $2 \ell 2 q \mathbb{E}_{T}$ & \\
0.03 & $\ell \tau 4 q$ & & 0.01 & $\ell 3 \tau \mathbb{E}_{T}$ & \\
0.03 & $\tau 2 q \mathbb{E}_{T}$ & & 0. & $3 \ell \tau \mathbb{E}_{T}$ & \\
0.02 & $\ell 4 q \mathbb{E}_{T}$ & & 0. & $2 \tau \mathbb{E}_{T}$ & \\
0.02 & $2 \ell 2 \tau \mathbb{E}_{T}$ & & 0. & $3 \ell 2 q \mathbb{E}_{T}$ & $\checkmark$ \\
0.02 & $2 \ell \tau 2 q \mathbb{E}_{T}$ & & 0. & $2 \ell \mathbb{E}_{T}$ & \\
0.02 & $2 q \mathbb{E}_{T}$ & & 0. & $4 \tau \mathbb{E}_{T}$ & \\
0.01 & $3 \tau 2 q \mathbb{E}_{T}$ & & 0. & $4 \ell \mathbb{E}_{T}$ & $\checkmark$ \\
0.01 & $\ell \tau \mathbb{E}_{T}$ & & & & \\
\hline
\end{tabular}

Table 3. Decay rates from $N N$ production for $\mathbf{B P} \mathbf{1}_{\mathbf{N H}}$ (left) and $\mathbf{B P} \mathbf{2}_{\mathbf{N H}}$ (right). The rates are obtained by summing on all charges and flavor configurations. Here $\ell=e, \mu$. The checkmarks correspond to channels that can produce a SS lepton signal.

\begin{tabular}{|c|c|c|c|c|c|}
\hline BR & Channel & SS & BR & Channel & SS \\
\hline 0.24 & $2 \ell 4 q$ & $\checkmark$ & 0.01 & $\ell \tau \mathbb{E}_{T}$ & \\
0.11 & $\ell 4 q \mathbb{E}_{T}$ & & 0.01 & $2 \ell 2 \tau \mathbb{E}_{T}$ & \\
0.07 & $3 \ell 2 q \mathbb{E}_{T}$ & $\checkmark$ & 0. & $\ell \tau 4 q$ & \\
0.05 & $4 q \mathbb{E}_{T}$ & & 0. & $\tau 4 q \mathbb{E}_{T}$ & \\
0.04 & $\ell 2 q \mathbb{E}_{T}$ & & 0. & $\ell 2 \tau 2 q \mathbb{E}_{T}$ & \\
0.04 & $2 \ell \tau 2 q \mathbb{E}_{T}$ & & 0. & $\tau 2 q \mathbb{E}_{T}$ & \\
0.03 & $2 \ell 2 q \mathbb{E}_{T}$ & & 0. & $2 \tau 2 q \mathbb{E}_{T}$ & \\
0.02 & $4 \ell \mathbb{E}_{T}$ & $\checkmark$ & 0. & $2 \tau \mathbb{E}_{T}$ & \\
0.02 & $2 q \mathbb{E}_{T}$ & & 0. & $\ell 3 \tau \mathbb{E}_{T}$ & \\
0.02 & $\ell \tau 2 q \mathbb{E}_{T}$ & & 0. & $2 \tau 4 q$ & \\
0.01 & $2 \ell \mathbb{E}_{T}$ & & 0. & $3 \tau 2 q \mathbb{E}_{T}$ & \\
0.01 & $3 \ell \tau \mathbb{E}_{T}$ & & 0. & $4 \tau \mathbb{E}_{T}$ & \\
0.01 & $\mathbb{E}_{T}$ & & & & \\
\hline
\end{tabular}

\begin{tabular}{|c|c|c|c|c|c|}
\hline BR & Channel & SS & BR & Channel & SS \\
\hline 0.06 & $2 \tau 4 q$ & & 0.01 & $2 \ell 2 q \mathbb{E}_{T}$ & \\
0.06 & $\tau 4 q \mathbb{E}_{T}$ & & 0.01 & $3 \ell 2 q \mathbb{E}_{T}$ & $\checkmark$ \\
0.06 & $4 q \mathbb{E}_{T}$ & & 0.01 & $\ell \tau \mathbb{E}_{T}$ & \\
0.05 & $\ell \tau 4 q$ & & 0.01 & $3 \ell \tau \mathbb{E}_{T}$ & \\
0.05 & $\ell 4 q \mathbb{E}_{T}$ & & 0.01 & $\mathbb{E}_{T}$ & \\
0.05 & $2 \ell 4 q$ & $\checkmark$ & 0.01 & $3 \tau 2 q \mathbb{E}_{T}$ & \\
0.04 & $2 \ell 2 \tau 2 q \mathbb{E}_{T}$ & & 0.01 & $2 \tau 2 q \mathbb{E}_{T}$ & \\
0.04 & $\ell 2 \tau 2 q \mathbb{E}_{T}$ & & 0.01 & $2 \ell \mathbb{E}_{T}$ & \\
0.03 & $\ell \tau 2 q \mathbb{E}_{T}$ & & 0 & $4 \ell \mathbb{E}_{T}$ & $\checkmark$ \\
0.02 & $2 q \tau \mathbb{E}_{T}$ & & 0 & $\ell 3 \tau \mathbb{E}_{T}$ & \\
0.02 & $2 q \mathbb{E}_{T}$ & & 0 & $2 \tau \mathbb{E}_{T}$ & \\
0.02 & $\ell 2 q \mathbb{E}_{T}$ & & 0 & $4 \tau \mathbb{E}_{T}$ & \\
0.02 & $2 \ell 2 \tau \mathbb{E}_{T}$ & & & & \\
\hline
\end{tabular}

Table 4. Decay rates from $N N$ production for $\mathbf{B P} \mathbf{1}_{\mathbf{I H}}$ (left) and $\mathbf{B P} \mathbf{2}_{\mathbf{I H}}$ (right). The rates are obtained by summing on all charges and flavor configurations. Here $\ell=e, \mu$. The checkmarks correspond to channels that can produce a SS lepton signal.

Prompt decay. We consider a RH neutrino decay as prompt if it happens within $\sim 0.1 \mathrm{~cm}$ from the primary vertex. The production of a pair of $N$ gives rise to a sixbody final state, including signatures with high lepton multiplicity, see table 3 and table 4. As we will see, in order to have promptly decaying RH neutrinos, one needs to have a large breaking of the naive see-saw scaling between the active-sterile mixing, the RH neutrino and the light neutrino masses. In the notation of section 2 this breaking is parametrized by a large value of the $\gamma$ parameter, see eq. (2.14). Large mixing angles are however constrained by a variety of experimental searches, and too large values of $\gamma$ are thus ruled out. 
Displaced decay. A particle is considered to decay displaced if it decays away from the primary vertex but within the detector environment. The precise distance for defining a vertex to be displaced clearly depends on the specific detector geometry. Given that our study focuses on future proposed $e^{+} e^{-}$colliders, for which detailed detector characteristics have not yet been settled, we consider as displaced particles decaying between $0.1 \mathrm{~cm}$ and $1 \mathrm{~m}$ from the primary vertex. Given the preliminary nature of our study we also consider the detector to have a spherical symmetry, instead of a cylindrical one.

Decays outside the detector. Also in this case the precise value of the decay length of the RH neutrinos in order for it to be considered detector stable depends on the specific geometry of the detector. We then consider as detector stable, RH neutrinos which decay more than $5 \mathrm{~m}$ away from the primary vertex.

\section{$3 \quad$ Future Higgs Factories}

\subsection{Collider prototypes}

Lepton colliders are ideal machines for SM precision measurements due to the cleanliness of their environment, the precise knowledge of the initial-state particles configuration and, for some prototypes, the possibility of having polarized beams that can help to enhance the signal-to-background ratio. Despite their center-of-mass energy being typically smaller than the one of hadronic machines, they however offer excellent prospects in the direct search for NP. This is mainly due to the low SM backgrounds, whose rates are generically comparable to the searched signals, as opposed to what happens in hadronic machines. Amongst the various proposals for a new generation of colliders after the HL-LHC era, leptons colliders then stand out as one of the more concrete possibility.

Various $e^{+} e^{-}$prototypes, presently at different stages of their design, have been proposed. These include circular ones, as the Future Circular Collider (FCC-ee) [34-37] and the Circular Electron Positron Collider (CEPC) [38, 39], and linear ones, as the International Linear Collider (ILC) [40-42] and the Compact Linear Collider (CLIC) [43, 44]. We report in table 5 the center-of-mass energies and luminosities considered in this study for various benchmark configurations. Notice that for CLIC we focus on its low-energy stage at $\sqrt{s}=380 \mathrm{GeV}$, to which we refer as CLIC-380 throughout the text. We report both the parameters for Higgs physics runs as well as the ones for runs at $\sqrt{s}=m_{Z}$. For what concerns the Higgs-strahlung cross sections $\sigma\left(e^{+} e^{-} \rightarrow Z h\right)$, in the cases of the ILC and CLIC-380, these are reported under the assumptions of a beam polarization fraction $\left(\mathcal{P}_{e^{-}}, \mathcal{P}_{e^{+}}\right)$of $(-80 \%,+30 \%)$ and $(-80 \%, 0 \%)$, respectively.

\subsection{Simulation framework}

In our analysis, signal events have been simulated at parton level by MadGraph5_aMC@NLO [54]. The events have then been analysed with the MadAnalysis5 package [55-57]. We consider our sensitivity estimates to be preliminary, not including any irreducible or reducible backgrounds. Nevertheless, we expect them to be not too far from a realistic lepton-collider sensitivity projection. For instance, an Higgs invariant-mass selection cut on the final states 
Higgs run

\begin{tabular}{|c|c|c|c|}
\hline Collider & $\sqrt{s}[\mathrm{GeV}]$ & $\int \mathcal{L}\left[\mathrm{ab}^{-1}\right]$ & $\sigma_{Z h}[\mathrm{fb}]$ \\
\hline FCC-ee & 240 & 5 & 193 \\
\hline ILC & 250 & $2(\mathrm{pol})$ & 297 \\
\hline CLIC-380 & 380 & $1(\mathrm{pol})$ & 133 \\
\hline CEPC & 240 & 5.6 & 193 \\
\hline
\end{tabular}

$Z$ pole run

\begin{tabular}{|c|c|c|c|}
\hline Collider & $\sqrt{s}[\mathrm{GeV}]$ & $\int \mathcal{L}\left[\mathrm{ab}^{-1}\right]$ & $N_{Z}$ \\
\hline FCC-ee & $m_{Z}$ & 150 & $6.5 \times 10^{12}$ \\
\hline CEPC & $m_{Z}$ & 16 & $6.9 \times 10^{11}$ \\
\hline
\end{tabular}

Table 5. Center-of-mass energies and total integrated luminosities for the various collider options considered in the analysis for Higgs runs (left) and $Z$ pole runs (right). For the Higgs runs we report the values of the Higgs-strahlung cross-section reported in [52], while for the $Z$ pole runs the number of expected $Z$ bosons produced with the corresponding integrated luminosity from [53].

featuring very high multiplicity Higgs decays, as we are considering here, should be sufficient for suppressing the irreducible SM backgrounds in the relatively clean lepton-collisions environment. Although a full simulations of the actual detector performance, when available, will certainly make the corresponding projections more robust, we are confident that, in a more realistic approach, the excellent accuracy of particle-flow reconstruction, as now under consideration for Higgs Factories detectors, complemented by advanced analysis techniques, might only moderately degrade the present sensitivity estimates.

\section{The $\mathcal{O}_{N H}$ operator and the Higgs-strahlung channel}

We start our analysis by discussing the $\mathcal{O}_{N H}$ operator. It can be generated by the tree-level exchange of a scalar singlet or by a fermion doublet with hypercharge $\pm 1 / 2$ [28]. It gives rise to a new interaction of the Higgs boson with a pair of $\mathrm{RH}$ neutrinos. If kinematically allowed, this interaction induces an extra decay channel for the Higgs boson, $h \rightarrow N N$. In the following we assume for simplicity degenerate RH neutrino masses $m_{N_{1}}=m_{N_{2}}=m_{N}$. For real couplings ${ }^{6}$ the partial width reads $[27]$

$$
\Gamma\left(h \rightarrow \bar{N}_{i}^{c} N_{i}\right)=\frac{1}{2 \pi} \frac{v^{2}}{\Lambda^{2}} m_{H} \beta_{N}^{3}\left(\alpha_{N H}^{i i}\right)^{2},
$$

where

$$
\beta_{N}=\sqrt{1-\frac{4 m_{N}^{2}}{m_{H}^{2}}} .
$$

This operator can be constrained by searches for additional untagged Higgs decay modes or invisible Higgs decays [52]. More importantly, it adds a new production mode for RH neutrinos through the Higgs-strahlung process

$$
e^{+} e^{-} \rightarrow Z h, h \rightarrow N N
$$

At lepton colliders the process of eq. (4.3) is the dominant production mode for a SM Higgs boson for center-of-mass energies $\sqrt{s} \lesssim 400 \mathrm{GeV}$. It is crucial that, by using the recoil

\footnotetext{
${ }^{6}$ For imaginary couplings there is a different dependence on the $\mathrm{RH}$ neutrino velocity $\beta_{N}$ due to $\mathrm{CP}$ properties of the matrix element see, e.g., [27].
} 
mass technique, this process can be tagged by reconstructing the $Z$ decay products, without any knowledge of the particles arising from the Higgs boson decay. This property makes this channel important for all the three regimes of the RH neutrinos lifetimes described in section 2.4. The Higgs-strahlung cross-sections for the various colliders are normalized as reported in table 5 .

\subsection{Prompt decay}

As anticipated in section 2.1, in order to have the RH neutrinos to decay promptly, one needs a large breaking of the naive see-saw scaling, parametrized by a large value of the parameter $\gamma$ entering the Casas-Ibarra parametrization of eq. (2.8) and enhancing the active-sterile mixing angle. This mixing is however constrained by a variety of experimental searches and too large values of $\gamma$ are ruled out. Using the bounds on $\theta_{\alpha}=\sum_{i=1,2}\left|\theta_{\alpha i}\right|^{2}$ reported in [58-60], we show in figure 2 as a gray dashed line the exclusion contour in the $m_{N}-\left|\theta_{e}^{2}\right|$ plane for the NH case. Similar results are obtained for IH. We show only the bound arising from $\theta_{\mu}$, which turns out to be the most stringent one. Notice that $\left|\theta_{e}\right|^{2}$ inherits the bound from $\theta_{\mu}$ through its dependence on $\gamma$ and $m_{N}$. In the same plot we also show as black dashed lines the isocontours of proper decay length $c \tau$ of the $\mathrm{RH}$ neutrino $N_{1}$, in order to identify the regions where the decay is prompt, displaced or outside of the detector. ${ }^{7}$ We neglect the dependence of the lifetime on $\beta, \delta$ and $\phi_{1}$ which is mild for $\gamma \gtrsim 1$. We also show the colored regions in which the RH neutrino pair production from the Higgs-strahlung process of eq. (4.3) is larger than the production of a single RH neutrinos via mixing. For concreteness we consider the case of the FCC-ee collider with $\sqrt{s}=240 \mathrm{GeV}$, and compare the Higgs-strahlung cross section with the one of production via mixing. Since the result depends on the branching ratio of the Higgs boson into RH states, we show our results for $\operatorname{BR}(h \rightarrow N N)=10 \%$ (red), $1 \%$ (blue) and $0.1 \%$ (green), using [18] for the normalization of the mixing cross-section. Finally, in the gray area at the bottom of the plot the lightness of the neutrino masses cannot be explained by the see-saw mechanism.

Altogether, the figure makes clear that there are large regions in parameter space in which Higgs-strahlung production may dominate over mixing production. Moreover, depending on $m_{N}$, in these regions the $\mathrm{RH}$ neutrinos can have prompt, displaced or outside the detector decays depending on the active-sterile mixing.

\subsubsection{Projected sensitivities: $e$ and $\mu$ mixing}

We start by considering the benchmark points which minimize the mixing with the third generation leptons, namely $\mathbf{B P} \mathbf{1}_{\mathbf{N H}}$ and $\mathbf{B P} \mathbf{1}_{\mathbf{I H}}$ of eq. (2.16) and eq. (2.17). For these two cases we focus for simplicity on the final state with the highest rate, which according to

\footnotetext{
${ }^{7}$ Strictly speaking, the important quantity is the laboratory frame decay length, which is larger than the proper decay length due to Lorentz time dilation. We will accurately compute this quantity in section 4.2 when dealing with displaced vertices while in this section we assume to be in a region of the $m_{N}-\left|\theta_{e}^{2}\right|$ parameter space where the Lorentz factor does not modify the behavior between prompt, displaced or stable. This is the case for $c \tau$ values away from the boundary regions indicated in the plot for not too light $N$.
} 


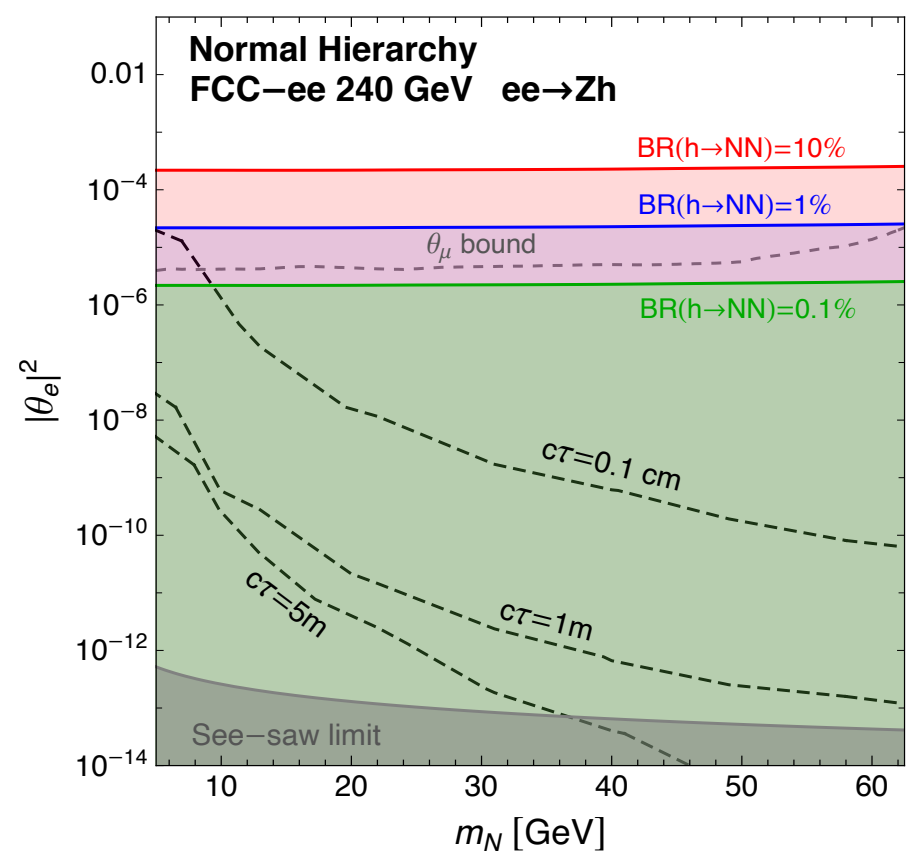

Figure 2. Regions in the $m_{N}-\left|\theta_{e}\right|^{2}$ parameter space where the RH neutrino pair production from the Higgs-strahlung process of eq. (4.3) is larger than the production of a single RH neutrinos via mixing, for $\mathrm{BR}(h \rightarrow N N)=10 \%$ (red), $1 \%$ (blue) and $0.1 \%$ (green) for the FCC-ee case. The NH case is assumed. The gray dashed line represents the limit on the mixing angle arising from existing experimental searches, while the black dashed lines represent isocontour of proper decay length $c \tau$. In the gray shaded region the lightness of the neutrino masses cannot be explained by the see-saw mechanism.

table 3 and table 4 is the $2 \ell 4 q$ one. Furthermore, we require this final state to contain a pair of SS leptons, thus halving the rates reported in the tables, and focus on the case in which the $Z$ boson decays leptonically. In computing our limits we sum on both $e$ and $\mu$ flavor combinations for the $Z$ and the $N$ pair decay modes, and on both the RH neutrinos $N_{1}$ and $N_{2}$. All together the process we analyze is

$$
e^{+} e^{-} \rightarrow Z h \rightarrow\left(\ell_{\alpha}^{+} \ell_{\alpha}^{-}\right)\left(\ell_{\beta}^{+} \ell_{\gamma}^{+} 4 q\right)+h . c .
$$

where the first bracket indicates the $Z$ boson decay products, while the second bracket the Higgs boson ones and $\alpha, \beta, \gamma=1,2$ are flavor indices. This is shown in figure 3. For our analysis we require the leptons to have $p_{T}>2.5 \mathrm{GeV}$ and $|\eta|<2.44$, while jets should satisfy $p_{T}>5 \mathrm{GeV}$ and $|\eta|<2.4$. Leptons are required to be separated by $\Delta R>0.15$ among themselves and with respect to the selected jets. In order to tag the Higgs-strahlung topology, we require two same-flavor opposite-sign leptons with an invariant mass $\mid m_{\ell^{+} \ell^{-}}-$ $m_{Z} \mid<10 \mathrm{GeV}$. If more than one pair that satisfies this condition is present, we choose the pair with an invariant mass closer to the $Z$ mass. Furthermore this pair is also required to have a recoil mass $m_{\text {rec }}$ within $10 \mathrm{GeV}$ of the true Higgs mass $m_{H}$, where

$$
m_{\mathrm{rec}}^{2}=s-2 \sqrt{s} E_{\ell^{+} \ell^{-}}+m_{\ell^{+} \ell^{-}}^{2},
$$




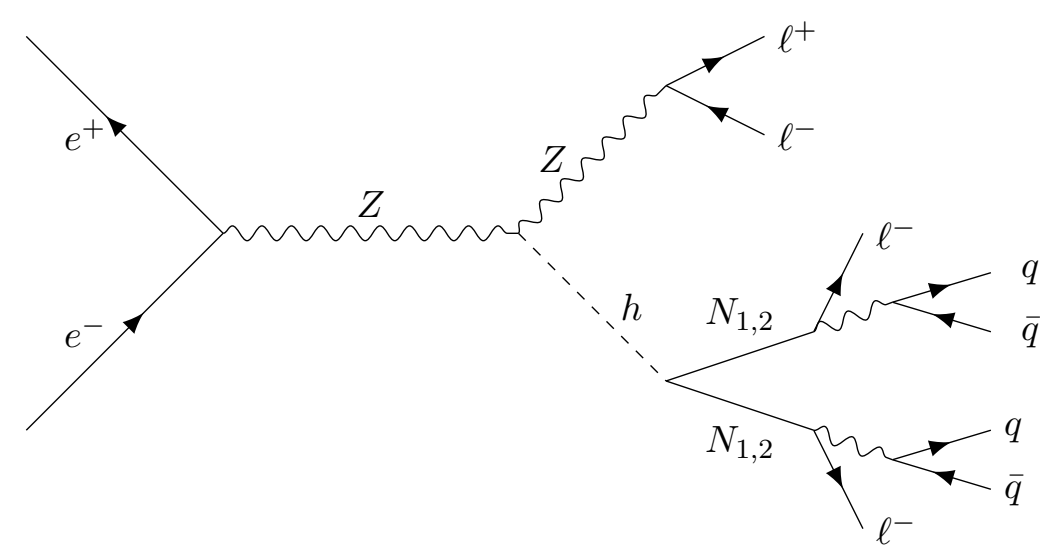

Figure 3. Feynman diagram for $2 \ell 4 q$ production in the SS leptons final state through Higgsstrahlung production.

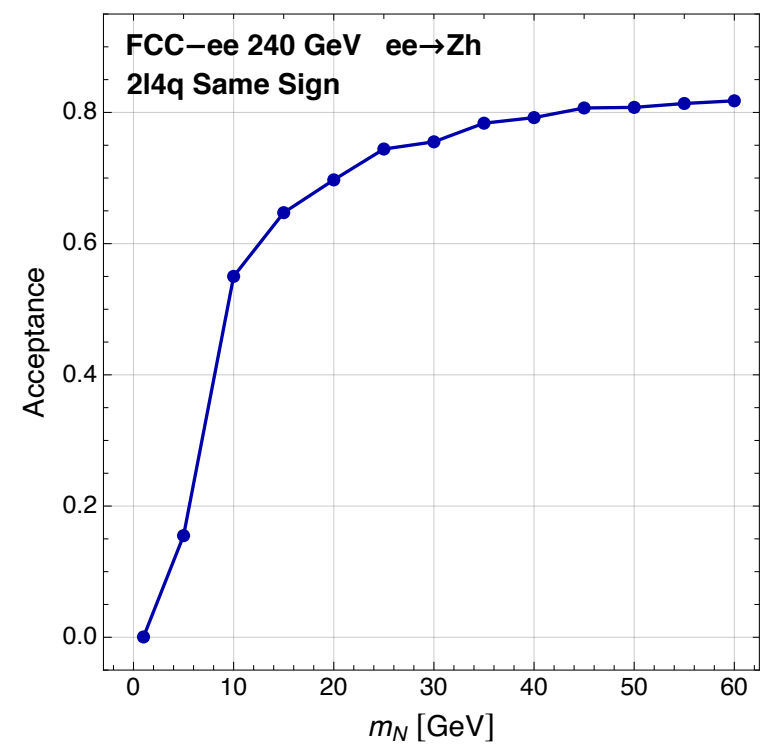

Figure 4. Parton level acceptances for the $2 \ell 4 q$ final state in the Higgs-strahlung topology with a leptonically decaying $Z$ boson and with the selection cuts described in the main text.

and $E_{\ell^{+} \ell^{-}}$is the energy of the leptons pair. We then ask for exactly two other SS leptons in the event, while no requirements on the number of jets and missing energy is imposed.

Three final state flavor configurations from the $N N$ decay contribute to the selected final state: $e^{+} e^{+}, e^{+} \mu^{+}$and $\mu^{+} \mu^{+}$plus the charge conjugated processes. The parton level acceptances as a function of the RH neutrino $\operatorname{mass}^{8}$ for these final states are shown in figure 4. As it can be seen the acceptances increase with the increase of the $\mathrm{RH}$ neutrino mass. This is to be expected, since for light $\mathrm{RH}$ neutrinos their decay products turn out to be more collimated, thus making it harder to pass the $\Delta R>0.15$ isolation criteria.

\footnotetext{
${ }^{8}$ The acceptances are computed for the fully electron final state. Similar acceptances are obtained for the other flavor combinations.
} 
Regarding the irreducible SM backgrounds to the process of eq. (4.4), we expect it to be negligible. In principle, since we are not requiring any jet in the final state, any SM process matching a signature $\left(Z \rightarrow \ell_{\alpha}^{+} \ell_{\alpha}^{-}\right)\left(h \rightarrow \ell_{\beta}^{+} \ell_{\gamma}^{+}+\ldots\right)$ could mimic the signal. Lepton-number conservation in the SM however implies that at least two extra neutrinos should be present in the final state. Electric-charge conservation moreover implies that there should also be at least two further quark pairs, arising e.g. from two off-shell $W$ 's. A typical SM irreducible background to the process under consideration is therefore

$$
e^{+} e^{-} \rightarrow\left(Z \rightarrow \ell_{\alpha}^{+} \ell_{\alpha}^{-}\right)\left(h \rightarrow \ell_{\beta}^{+} \nu_{\beta} \ell_{\gamma}^{+} \nu_{\gamma} \bar{u} d \bar{u} d\right)
$$

where the final state arising from the Higgs decay involves multiple off-shell gauge bosons, such a $h \rightarrow 4 W^{*}$ decay. We then expect it to be totally negligible. Due to the very large multiplicity of the chosen signal, we also expect other channels that match the same final state without occurring via $Z$ and Higgs resonances to be negligible. As for other reducible backgrounds arising from the limited efficiencies in the detector performances, such as e.g. mis-identification of the final particles, we are confident that they can be efficiently removed thanks to the strong kinematical characterization of the $\left(Z \rightarrow \ell_{\alpha}^{+} \ell_{\alpha}^{-}\right)\left(h \rightarrow \ell_{\beta}^{+} \ell_{\gamma}^{+}+\ldots\right)$ process. We thus assume the process in eq. (4.4) to be background free. According to Poisson statistics, in case no signal event is observed, one can then set a $95 \%$ confidencelevel (CL) exclusion limit corresponding to a maximally allowed number $N_{s}=3$ of new physics events [61].

We show our results for $\mathbf{B P} \mathbf{1}_{\mathbf{N H}}$ in the left panel of figure 5 . Similar results are obtained for the $\mathbf{B P} \mathbf{1}_{\mathbf{I H}}$ benchmark. The colored regions represent the $95 \%$ CL sensitivity of the analysis on the exotic Higgs BR described above in the $m_{N}-\mathrm{BR}(h \rightarrow N N)$ plane for the various collider options. This reach has to be compared with the limits coming from untagged Higgs decay [52], represented by horizontal dashed colored lines. For simplicity we do not show the limit that might be obtained at CEPC, which are comparable to the ones from FCC-ee, due to their similar integrated luminosity, see table 5. We also show in dashed black the isocontours of the scale $\Lambda$ expressed in $\mathrm{TeV}$, fixing $\alpha_{N H}^{i i}=1$. Finally, we highlight in gray the region where $c \tau>1 \mathrm{~mm}$ in which the $\mathrm{RH}$ neutrino cannot decay promptly without being excluded by experimental searches, see figure 2 .

All together we see that future $e^{+} e^{-}$colliders will be able to set a bound on the exotic Higgs BR that goes from $\sim 5 \times 10^{-3}$ for the case of CLIC-380 down to $\sim 7 \times 10^{-4}$ for the case of FCC-ee/CEPC, and that these bounds are significantly stronger than the corresponding ones arising from untagged Higgs decay measurements. In terms of NP scale $\Lambda$ these limits translate in a bound which, in the most favorable case, is $\Lambda \gtrsim 500 \mathrm{TeV}$.

\subsubsection{Projected sensitivities: $\tau$ mixing}

We now consider the case where the active-sterile mixing with the third generation of leptons is maximized, as is the case for the benchmarks $\mathbf{B P} \mathbf{2}_{\mathbf{N H}}$ and $\mathbf{B P} \mathbf{2}_{\mathbf{I H}}$ in eq. (2.16) and eq. (2.17). Again we choose for simplicity the channel with the highest BR for both benchmark points, i.e. the $2 \tau 4 q$ channel. In this case however the $\tau$ leptons will promptly decay into a $\nu_{\tau}$ and an off-shell $W$ boson. For our analysis we adopt the following strategy. We first consider the $\tau$ leptons as stable particles, and apply the same parton-level 

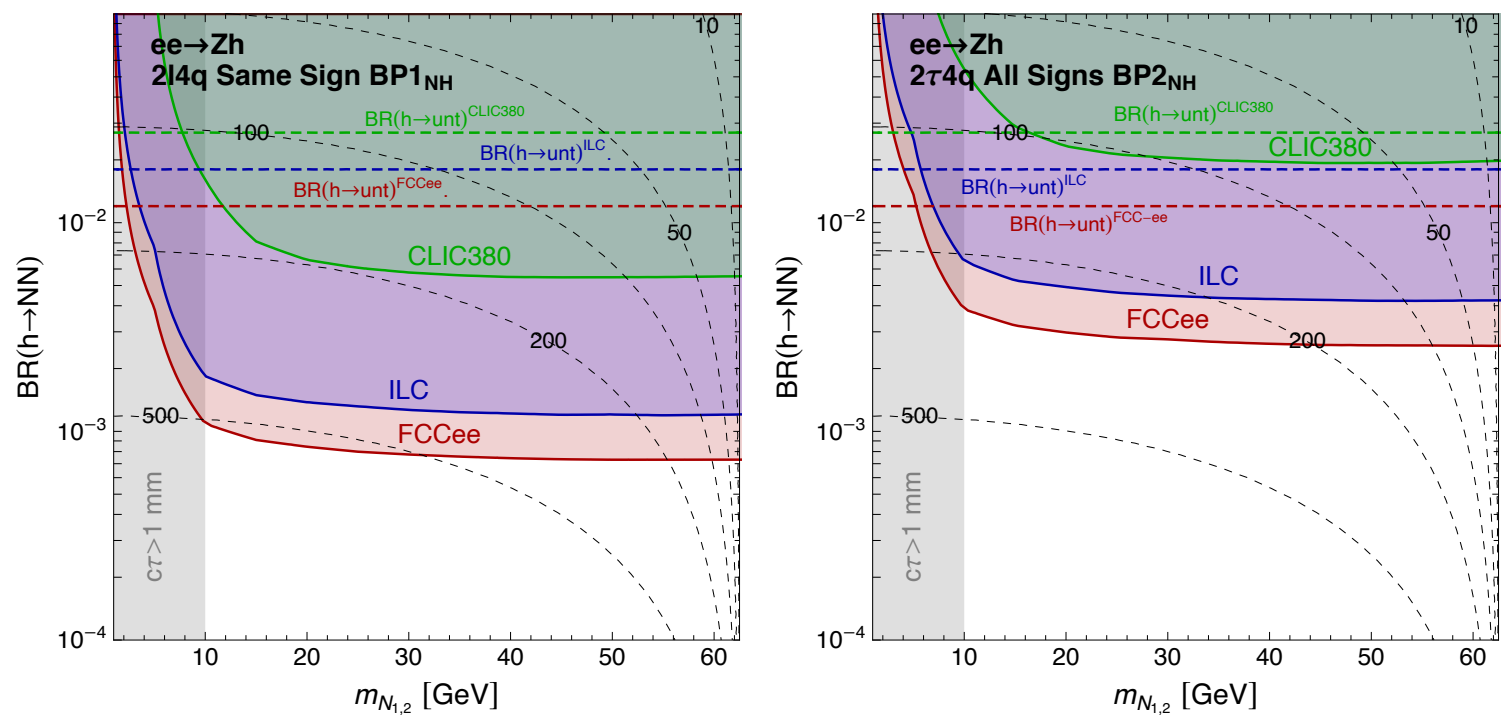

Figure 5. 95\% CL exclusion for $\mathbf{B P} \mathbf{1}_{\mathbf{N H}}$ (left) and $\mathbf{B P} \mathbf{2}_{\mathbf{N H}}$ (right) from prompt searches in the Higgs-strahlung channel. The shaded areas represent the exclusion contours for the case of FCC-ee (red), ILC (blue) and CLIC-380 (green) adopting the strategy of section 4.1.2 and section 4.1.2 for the case of the $2 \ell 4 q(\mathrm{SS})$ and $2 \tau 4 q$ final states respectively. Also shown as colored dashed lines the limits arising from untagged Higgs decay measurements and as gray dashed lines the isocontours of NP scale $\Lambda$ in $\mathrm{TeV}$. In the gray shaded area $c \tau>1 \mathrm{~mm}$ and the $\mathrm{RH}$ neutrino cannot decay promptly without being excluded by experimental searches.

selection efficiencies computed in the $e$ and $\mu$ case of section 4.1.1. Then we focus on the hadronic decay modes $\tau^{-} \rightarrow \pi^{-} \pi^{0} \nu_{\tau}, \tau^{-} \rightarrow \pi^{-} \nu_{\tau}$ and $\tau^{-} \rightarrow \pi^{-} \pi^{0} \pi^{0} \nu_{\tau}$ (which account, respectively, for approximately $25.5 \%, 10.8 \%$ and $9.3 \%$ of the total $\tau$ branching ratio) and apply, following [62], a flat $90 \%$ reconstruction efficiency for each $\tau$ lepton. We consider the inclusive $2 \tau 4 q$ final state with all charge combinations, $\tau^{ \pm} \tau^{ \pm}$and $\tau^{ \pm} \tau^{\mp}$, and, again, neglect the SM background, although in this case we expect a larger contamination with respect to the $2 \ell 4 q$ SS case. ${ }^{9}$ Under these assumptions we obtain the results shown in the right panel of figure 5 for $\mathbf{B P 2} \mathbf{2}_{\mathbf{N H}}$. Similar, albeit slightly weaker, results are obtained for the $\mathbf{B P 2} \mathbf{I H}_{\mathbf{I H}}$ case. We see that also in the case in which $N$ mixes dominantly with $\nu_{\tau}$, both FCC-ee and ILC can probe values of $\mathrm{BR}(h \rightarrow N N)$ that go well beyond the limits arising from untagged Higgs decay, while CLIC-380 can marginally surpass this reach.

\subsubsection{Determination of the flavor structure}

Should a RH neutrino signal be detected, a crucial question to be asked is with which accuracy it will be possible to determine the flavor structure of the underlying BSM theory. This is directly related to the number of signal events which in turn depends on the physics of the underlying theory, namely $\mathrm{BR}(h \rightarrow N N)$, and the detector performances in reconstructing the signal. To quantify this we adopt the following strategy. We choose the

\footnotetext{
${ }^{9}$ For instance we estimated the cross section for the irreducible background $e^{+} e^{-} \rightarrow\left(Z \rightarrow \mu^{+} \mu^{-}\right)(h \rightarrow$ $\left.\tau^{+} \tau^{-} u \bar{d} \bar{u} d\right)$ to be about $5.4 \times 10^{-6} \mathrm{pb}$, corresponding to about 27 events at FCC-ee. We expect that these can be efficiently reduced by exploiting the kinematical features of the $h \rightarrow N N$ decay.
} 

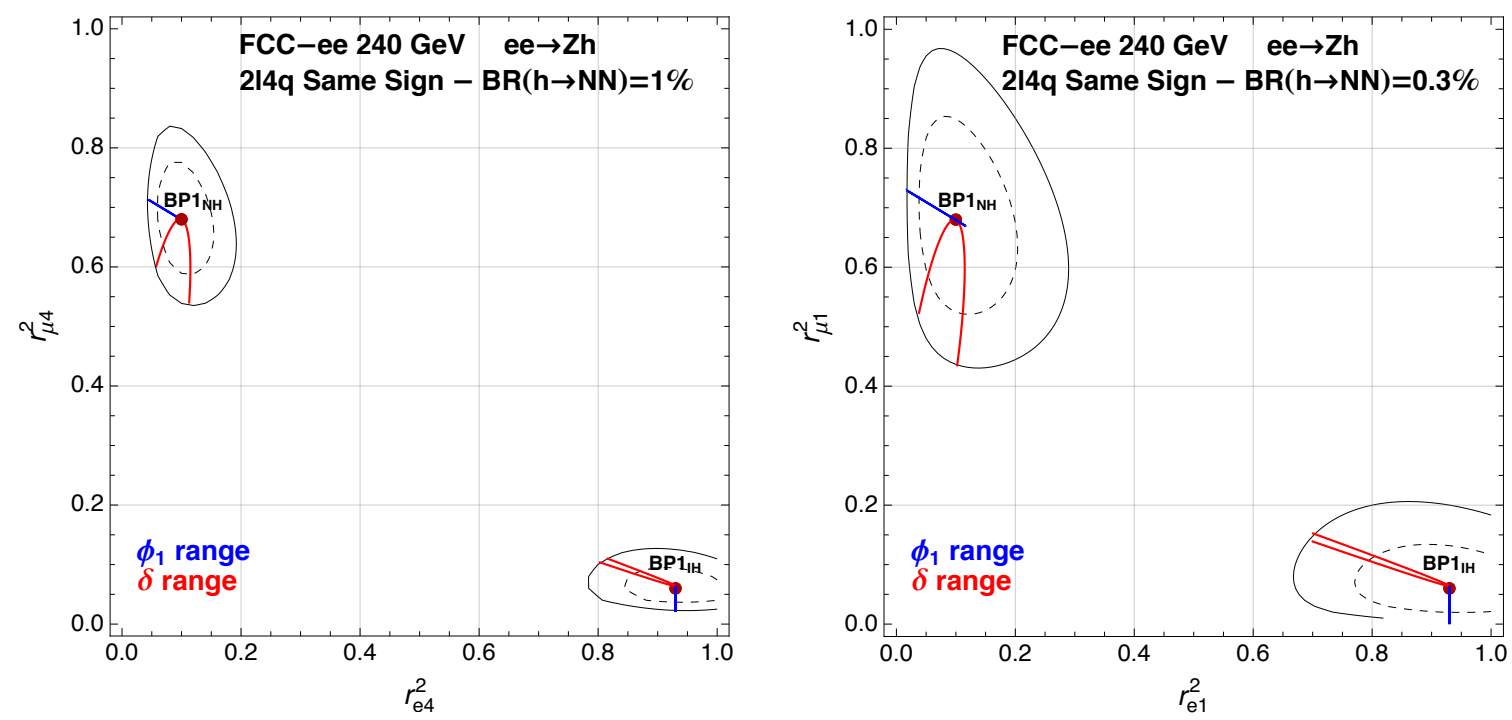

Figure 6. $68 \%$ (dashed) and $95 \%$ (solid) confidence intervals for determining the flavor structure of a putative BSM signal observed associated with $\mathbf{B P} \mathbf{1}_{\mathbf{N H}}$ and $\mathbf{B P} \mathbf{1}_{\mathbf{I H}}$ in the $2 \ell 4 q$ final state with a pair of SS leptons. We fix $\operatorname{BR}(h \rightarrow N N)=1 \%$ (left), $0.3 \%$ (right) and $m_{N}=30 \mathrm{GeV}$.

$2 \ell 4 q$ final state with a pair of SS leptons, a representative $\mathrm{RH}$ neutrino mass of $30 \mathrm{GeV}$ and the benchmark points $\mathbf{B P} \mathbf{1}_{\mathbf{N H}}$ and $\mathbf{B P} \mathbf{1}_{\mathbf{I H}}$. We then build a Poisson distribution $p\left(n_{\mathrm{obs}} \mid n_{\mathrm{th}}\right)=\frac{1}{n_{\mathrm{obs} !}} e^{-n_{\mathrm{th}}} n_{\mathrm{th}}^{n_{\mathrm{obs}}}$ where $n_{\mathrm{obs}}$ correspond to the expected number of observed events in the case of the presence of a BSM signal, and $n_{\text {th }}$ is the theoretical prediction for the number of events in the chosen final state, which is a function of $r_{e 1}^{2}$ and $r_{\mu 1}^{2}$. For fixed values of $\mathrm{BR}(h \rightarrow N N)$, which then fixes the final event yield, we thus compute the $68 \%$ and $95 \%$ CL confidence interval around the chosen benchmark points. The results are illustrated in figure 6 for the case of $\mathrm{BR}(h \rightarrow N N)=1 \%$ (left) and $0.3 \%$ (right) for the FCC-ee collider option. We see that with a $1 \%$ Higgs exotic BR, which correspond to $\sim 37$ total signal events in the final state, the normalized squared mixings $r_{e 1}$ and $r_{\mu 1}$ can be determined with an absolute error of $\sim 0.1$, which rapidly degrades down to $\sim 0.3$ with a $0.3 \%$ Higgs BR into a pair of $\mathrm{RH}$ neutrinos, for which one has $\sim 11$ signal events. It is interesting to interpret these results in terms of the phases appearing in the PMNS matrix, $\delta$ and $\phi_{1}$. The benchmark points we choose correspond to fixed values for both phases: $\left(\delta, \phi_{1}\right)=(0.76,4.59)$ for the $\mathbf{B P} \mathbf{1}_{\mathbf{N H}}$ benchmark, and $\left(\delta, \phi_{1}\right)=(3.25,1.60)$ for the $\mathbf{B P} \mathbf{1}_{\mathbf{I H}}$ benchmark. By keeping $\delta$ fixed and allowing $\phi_{1}$ to vary we obtain the blue lines in figure 6 , while by keeping $\phi_{1}$ fixed and allowing $\delta$ to vary we obtain the red lines. The interval that $\delta$ and $\phi_{1}$ can span inside the $95 \%$ CL confidence intervals around the benchmark points values are reported in table 6 .

\subsection{Displaced decay}

We now study the sensitivity for RH neutrinos decaying with a displacement which, as discussed in section 2.4, we take to be between $1 \mathrm{~cm}$ and $100 \mathrm{~cm}$ from the primary vertex, see figure 2. We consider decays into first and second generation leptons and we focus 


\begin{tabular}{|c|c|c|}
\hline & $\mathrm{BR}(h \rightarrow N N)=1 \%$ & $\mathrm{BR}(h \rightarrow N N)=0.3 \%$ \\
\hline \multirow{2}{*}{$\mathbf{B P} 1_{\mathbf{N H}}$} & $3.69 \leq \phi_{1} \leq 5.57$ & $0.037 \leq \phi_{1} \leq 5.95$ \\
& $0.78 \leq \delta \leq 1.85 \cup 4.47 \leq \delta \leq 5.55$ & $0 \leq \delta \leq 2.53 \cup 3.80 \leq \delta \leq 2 \pi$ \\
\hline \multirow{2}{*}{$\mathbf{B P} 1_{\mathbf{I H}}$} & $0.80 \leq \phi_{1} \leq 2.31$ & $0.51 \leq \phi_{1} \leq 2.31$ \\
& $1.33 \leq \delta \leq 5.09$ & $0 \leq \delta \leq 2 \pi$ \\
\hline
\end{tabular}

Table 6. Range of parameters that can be probed in case of detection of a RH neutrino decay for the representative $m_{N}$ mass and $\mathrm{BR}(h \rightarrow N N)$ considered in figure 6 . The range for $\phi_{1}$ corresponds to the red lines in the figure, while the range for $\delta$ to the blue ones. In both case the other phase is kept fixed to the benchmark point value.

again on the $2 \ell 4 q$ final state, which is the one that maximizes the decay rate of the $N N$ pair. The final state therefore consists of two prompt same flavor opposite sign leptons from the $Z$ boson decay and the $2 \ell 4 q$ system. The final event yield is given by

$N_{s}=\sigma_{Z h} \times \mathrm{BR}\left(Z \rightarrow \ell^{+} \ell^{-}\right) \times \mathrm{BR}(h \rightarrow N N) \times \mathrm{BR}(N N \rightarrow 2 \ell 4 q) \times \epsilon_{Z h} \times \epsilon_{P_{\Delta L}}^{2} \times \epsilon_{\text {disp. }}^{2} \times \mathcal{L}$,

where $\epsilon_{Z h}$ is the acceptance for reconstructing the $Z$ boson and the Higgs recoil mass from the two same flavor opposite sign prompt leptons. The parameter $\epsilon_{P_{\Delta L}}^{2}$ represents instead the acceptance for having both neutrinos decaying within a certain displacement from the primary vertex. This probability can be computed from the exponential decay law, taking into account the time dilation factor obtained by boosting the events from the $\mathrm{RH}$ neutrino rest frame to the laboratory frame. In practice we have computed, for each event and for each RH neutrino, the Lorentz $\beta$ and $\gamma$ factors

$$
\beta=\sqrt{1-\frac{1}{\gamma^{2}}}, \quad \gamma=\sqrt{1+\frac{|\vec{p}|^{2}}{M_{N}^{2}}},
$$

and assigned a probability for having the $\mathrm{RH}$ neutrino decaying at a distance $\Delta x=x_{f}-x_{i}$

$$
\mathcal{P}\left(x_{i}, x_{f}\right)=e^{-\frac{x_{i}}{\beta \gamma c \tau}}-e^{-\frac{x_{f}}{\beta \gamma c \tau}} .
$$

We have then accepted events where both RH neutrino decays happened between $1 \mathrm{~cm}$ and $100 \mathrm{~cm}$ from the primary vertex. Finally, with $\epsilon_{\text {disp. }}$ we parametrize the acceptance for reconstructing the displaced decay, including various detector inefficiencies, which will depend on the actual detector design and performances, and which therefore we assume as a free extra parameter in the analysis. The irreducible SM background is expected to be negligible on the considered decay lenghts.

We show in figure 7 the FCC-ee reach for the $\mathbf{B P} \mathbf{1}_{\mathbf{I H}}$ benchmark in the $m_{N}-\left|\theta_{e}\right|^{2}$ plane. In the left panel we fix $\operatorname{BR}(h \rightarrow N N)=1 \%$, while in the right panel we fix $\mathrm{BR}(h \rightarrow N N)=0.3 \%$. We show our results for two different efficiencies $\epsilon_{\text {disp. }}$ as reported in the plots. In both figures the gray shaded area represents the see-saw limit, while the values of the mixing angles above the gray dashed line are excluded by experimental searches, see the discussion in section 4.1. Our results show that the search for $\mathrm{RH}$ neutrinos arising 

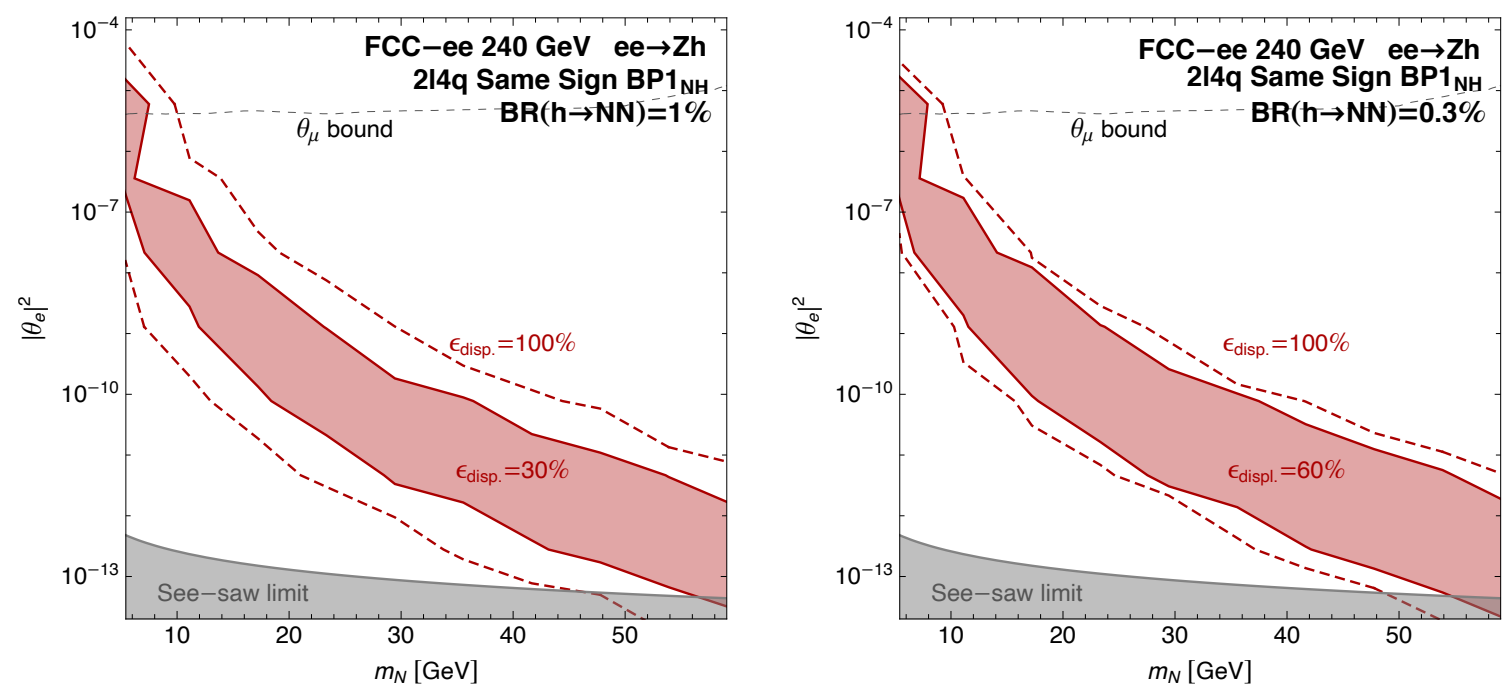

Figure 7. $95 \%$ CL exclusion for $\mathbf{B P} \mathbf{1}_{\mathbf{N H}}$ in the $m_{N}-\left|\theta_{e}\right|^{2}$ plane from displaced decay searches in the Higgs-strahlung channel assuming $\operatorname{BR}(h \rightarrow N N)=1 \%$ (left) and $0.3 \%$ (right) for the case of FCC-ee. The shaded red area and the dashed red lines represent the sensitivities for different assumptions on the efficiencies for reconstructing the displaced vertices $\epsilon_{\text {disp. }}$. The gray dashed line represents the limit on the mixing angle arising from existing experimental searches. In the gray shaded region the lightness of the neutrino masses cannot be explained by the see-saw mechanism.

from Higgs decay and decaying displaced can offer a great handle in testing the activesterile mixing angle. This is clearly due to the fact that, unlike in the case of production via mixing, the Higgs-strahlung cross section does not depend on the active-sterile mixing angle, which only enters in the lifetime determination, but only on the additional Higgs decay rate into an $N N$ pair. As a consequence, searches at FCC-ee could test values of $\left|\theta_{e}\right|^{2}$ down to the see-saw limit. We do not show for simplicity the limits arising from ILC and CEPC, which are comparable to the ones shown, and the one from CLIC-380, which turns out to be slightly weaker.

In figure 8 we show instead the reach of the same search projected on the plane $\mathrm{BR}(h \rightarrow N N)$ versus $\frac{m_{h}}{2 m_{N}} c \tau$, which is roughly the laboratory-frame decay length of the RH neutrinos. We do this again for two different choices of $\epsilon_{\text {disp. }}: 50 \%$ (left) and $100 \%$ (right). In the plots we also show the limits arising from untagged Higgs decays searches for the various collider options. All together we see that FCC-ee will be able to test values of the Higgs exotic BR down to $0.2 \%$ for $\epsilon_{\text {disp. }}=50 \%$, largely surpassing the indirect limits from Higgs untagged decays, while ILC and CLIC-380 have a slightly weaker reach.

\subsection{Detector stable}

The last case we study is the one in which the $\mathrm{RH}$ neutrinos lifetime is large enough that they will decay outside the detector. In this case the decay will contribute to the invisible Higgs width. This quantity can be strongly constrained at future lepton colliders, which will set a $95 \%$ CL bound on $\mathrm{BR}(h \rightarrow$ inv.) of $0.22 \%$ (FCC-ee), $0.28 \%$ (CEPC), $0.26 \%$ (ILC) and $0.63 \%$ (CLIC-380) [52]. These limits can be directly translated on a bound on 

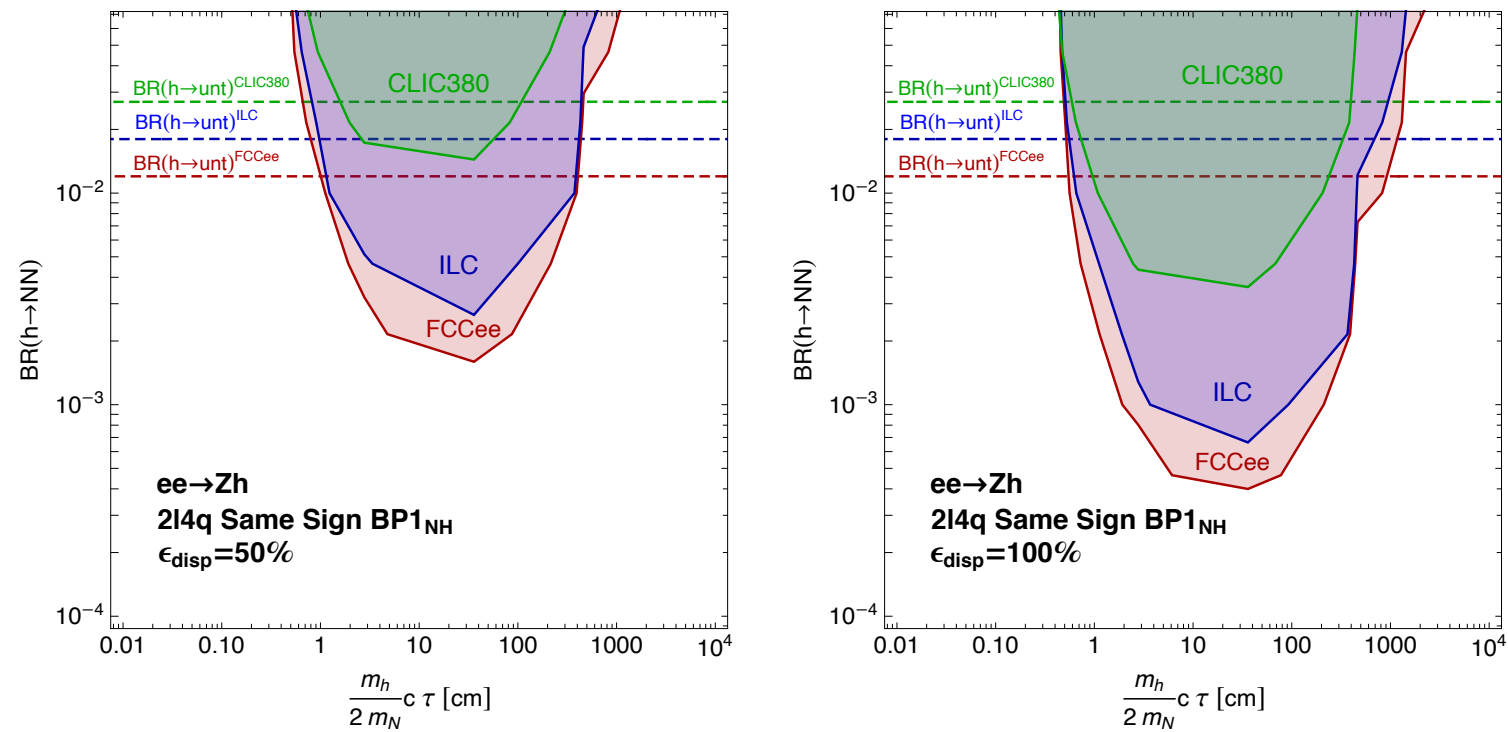

Figure 8. $95 \% \mathrm{CL}$ exclusion for $\mathbf{B P} \mathbf{1}_{\mathbf{N H}}$ in the $\frac{m_{h}}{2 m_{N}} c \tau-\mathrm{BR}(h \rightarrow N N)$ plane from displaced decay searches in the Higgs-strahlung channel assuming $\epsilon_{\text {disp. }}=50 \%$ (left) and $100 \%$ (right). The shaded red areas represent the sensitivities for the different collider options while the colored dashed lines the limits arising from untagged Higgs decay measurements.

the NP scale $\Lambda$ through eq. (4.1). We obtain $\Lambda \gtrsim 360 \mathrm{TeV}$ (FCC-ee), $320 \mathrm{TeV}$ (CEPC), $\Lambda \gtrsim 330 \mathrm{TeV}$ (ILC) and $210 \mathrm{TeV}$ (CLIC-380) fixing $m_{N}=10 \mathrm{GeV}$. The limits degrade by roughly a factor $20 \%$ for $m_{N}=35 \mathrm{GeV}$ due to the reduced phase space. Above this mass threshold their decay will instead happen inside the detector, see figure 2.

\section{The $\mathcal{O}_{N B}$ operator and the s-channel $Z$ production}

We turn now to the study of the $\mathcal{O}_{N B}$ dipole operator. It can be generated only a looplevel by a scalar-fermion of vector-fermion pair with opposite hypercharges [28]. Due to the presence of $\sigma^{\mu \nu}$, the flavor structure of this operator is antisymmetric, i.e. only different $\mathrm{RH}$ neutrinos can participate in the interaction. Since the operator induces a new interaction between the RH neutrinos and the SM neutral EW gauge bosons, it may provide an additional production channel for a pair of $\mathrm{RH}$ neutrinos through an intermediate photon or $Z$ boson. In particular, if kinematically allowed, the $Z$ boson can decay into a $N_{1} N_{2}$ pair with a rate $[28]$

$$
\Gamma_{Z \rightarrow N_{1} N_{2}}=\frac{2}{3 \pi} \frac{\left|\alpha_{N B}^{12}\right|^{2}}{\Lambda^{2}} \frac{s_{w}^{2}}{m_{Z}^{3}} \lambda^{1 / 2}\left(m_{Z}^{2}, m_{N_{1}}^{2}, m_{N_{2}}^{2}\right) \zeta\left(m_{Z}, m_{N_{1}}, m_{N_{2}}\right)
$$

where $s_{w}$ is the sine of the Weinberg angle, $\lambda(a, b, c)=a^{2}+b^{2}+c^{2}-2 a b-2 b c-2 a c$ and

$$
\zeta\left(m_{Z}, m_{N_{1}}, m_{N_{2}}\right)=m_{Z}^{2}\left(m_{Z}^{2}+m_{N_{1}}^{2}+m_{N_{2}}^{2}-6 m_{N_{1}} m_{N_{2}} \cos 2 \phi_{12}\right)-2\left(m_{N_{1}}^{2}-m_{N_{2}}^{2}\right)^{2},
$$

with $\phi_{12}=\arg \left[\alpha_{N B}^{12}\right]$. In the following we will fix $\phi_{12}=0$, i.e. we assume real Wilson coefficients. 

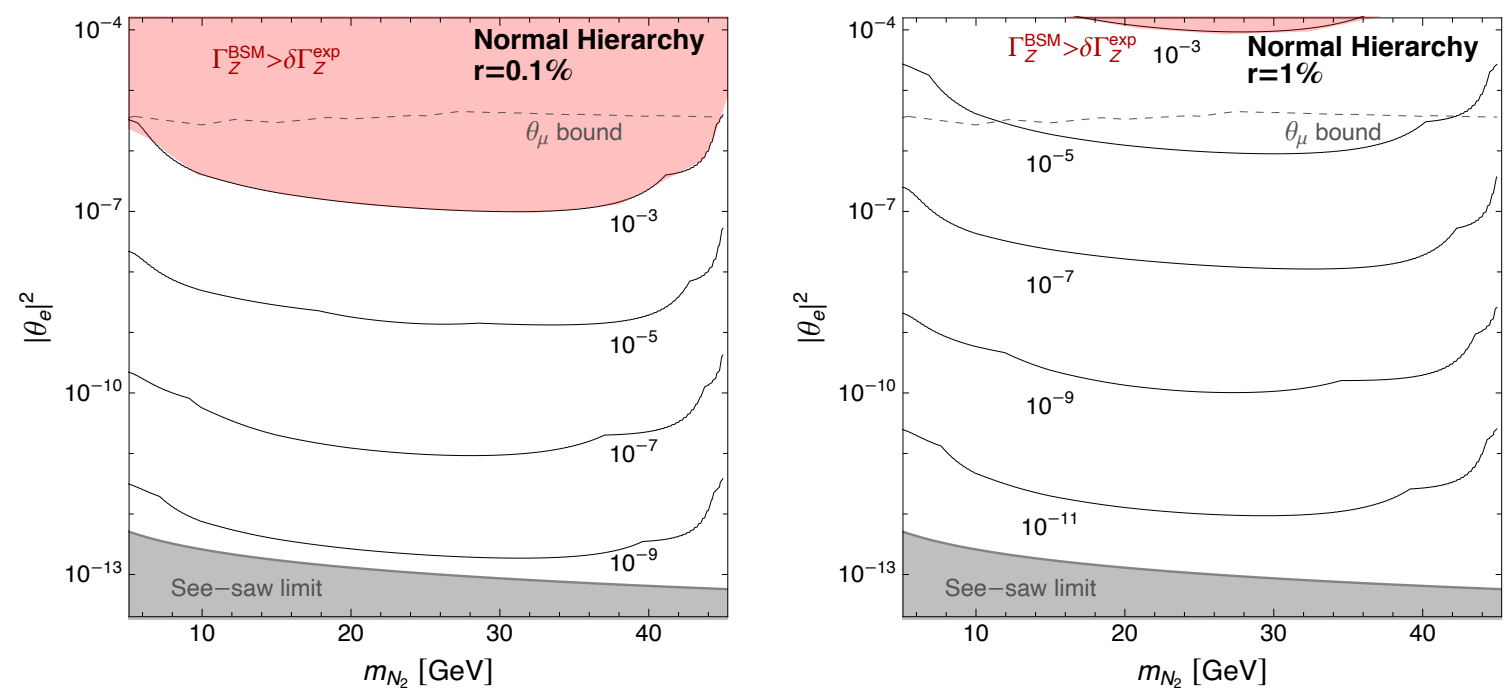

Figure 9. Isocontour of equal partial widths for $N_{2} \rightarrow N_{1} \gamma$ and $N_{2}$ decaying via mixing for various choices of $\operatorname{BR}\left(Z \rightarrow N_{1} N_{2}\right)$. We fix the relative mass splitting between $N_{2}$ and $N_{1}$ to $0.1 \%$ (left) and $1 \%$ (right). Above the lines, for that specific $\operatorname{BR}\left(Z \rightarrow N_{1} N_{2}\right)$, the decay via mixing dominates. In the red shaded area $\Gamma_{Z}^{\mathrm{BSM}}$ is larger than the current experimental uncertainty on the $Z$ boson total width. The gray dashed line represents the limit on the mixing angle arising from existing experimental searches. In the gray shaded region the lightness of the neutrino masses cannot be explained by the see-saw mechanism.

Future colliders with an operating stage at $\sqrt{s}=m_{Z}$, as is the case of FCC-ee and CEPC, will produce a large number of $Z$ bosons, see table 5 , and can thus probe the operator responsible for the decay of eq. (5.1) with high precision. We focus in particular on the case of FCC-ee, where one expects to have $6.5 \times 10^{12} Z$ candidates produced, after a total integrated luminosity of $\sim 150 \mathrm{ab}^{-1}$, while CEPC might have a luminosity smaller by roughly one order of magnitude, and weaker results are generally expected.

In addition to new production modes, the $\mathcal{O}_{N B}$ operator can also trigger new decay channels for the heavier $\mathrm{RH}$ neutrino $N_{2}$. In particular we have [28]

$$
\Gamma\left(N_{2} \rightarrow N_{1} \gamma\right)=\frac{2}{\pi} c_{w}^{2} \frac{\left|\alpha_{N B}\right|^{2}}{\Lambda^{2}} m_{N_{2}}^{3}\left(1-\frac{m_{N_{1}}^{2}}{m_{N_{2}}^{2}}\right)^{3},
$$

while we do not consider the possibility of $N_{2} \rightarrow N_{1} Z$ decay which is outside of the mass range of interest here. Crucially, the decay mode of eq. (5.3) can compete, and even dominate, with the one induced by the mixing between the active and sterile sector. It is thus important to assess in which region of parameter space the $N_{2} \rightarrow N_{1} \gamma$ decay rate can dominate over the decay induced by active-sterile mixing. In figure 9 we show such regions in the $m_{N_{1}}-\left|\theta_{e}\right|^{2}$ plane considering the $\mathrm{NH}$ case. As in previous plots, we show the limits coming from $\theta_{\mu}$ as gray dashed line. The black continuous lines correspond to $\Gamma\left(N_{2} \rightarrow N_{1} \gamma\right)=\Gamma\left(N_{2}\right)_{\text {mix }}$, where the latter is the total $N_{2}$ decay width due to mixing, i.e. in the channels listed in table 1 , for the specific value of $\operatorname{BR}\left(Z \rightarrow N_{1} N_{2}\right)$ reported. Above the lines the decays induced by mixing dominate over the decay induced by the $\mathcal{O}_{N B}$ 


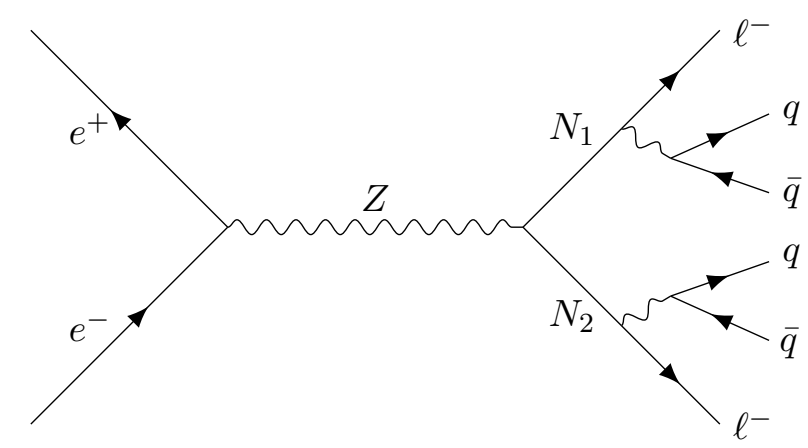

Figure 10. Feynman diagram for $2 \ell 4 q$ production in the SS leptons final state through s-channel $Z$ production.

operator. Since this operator involves different neutrinos, different mass splittings may give different physical situations. We quantify this by defining the relative mass splitting $r=\left(m_{N_{2}}-m_{N_{1}}\right) / m_{N_{2}}$, which is fixed to $0.1 \%$ in the left plot and to $1 \%$ in the right plot. We also show in red the region in which the total BSM width $\Gamma_{Z}^{\mathrm{BSM}}$ is larger than the than the current experimental uncertainty on the $Z$ boson total width, $\delta \Gamma_{Z}^{\exp }=2.3 \mathrm{MeV}$, and the region in which the see-saw mechanism is not able to reproduce the observed neutrino masses. As we see, in both cases for reasonable values of the branching ratio there are large regions in parameter space in which the decay via mixing dominates over $N_{2} \rightarrow N_{1} \gamma$.

As a consequence, in this section we will focus on the signatures of $N_{2}$ decaying via mixing. Notice that, barring the contribution of $d=6$ operators on which we will comment in section 6 , this is always true also for $N_{1}$. As it happened in section 4 , we find that for mixing angles compatible with experimental bounds, the $N_{1} N_{2}$ production via $Z$ decay can easily dominate with respect to the $N \nu$ production via mixing. In the following sections we will thus analyze the sensitivity of future experiments for the case in which the $N_{1} N_{2}$ production occurs via $Z$ decay and $N_{1,2}$ decay via mixing. As already discussed, also in this case the decay can be prompt, displaced or outside the detector. For simplicity we fix $m_{N_{2}}-m_{N_{1}} \ll m_{N_{1,2}}$, i.e. we study the case of almost degenerate $\mathrm{RH}$ neutrinos.

\subsection{Prompt decay}

We again focus on the final state with the highest rate, i.e. the $2 \ell 4 q$ channel, restricting our analysis to the final state with a pair of SS leptons. This process is shown in figure 10. We require exactly two SS leptons with $p_{T}>2.5 \mathrm{GeV}$ and $|\eta|<2.44$, while jets should satisfy $p_{T}>5 \mathrm{GeV}$ and $|\eta|<2.4$. Leptons are required to be separated by $\Delta R>0.15$ among themselves and with respect to the selected jets. With these selections, the parton level acceptances for the $e^{+} e^{+}$flavor combination are reported in figure 11.

Regarding the background analysis, in the case of the $\mathcal{O}_{N H}$ operator we assumed that the physical background mimicking the Higgs-stralung production and subsequent six-body decay in a sample of around $10^{6}$ Higgs bosons would be well under control and negligible in first approximation. On the other hand, in case of the FCC-ee production of $\mathcal{O}\left(10^{12}\right)$ $Z$ bosons at the $Z$ peak, quite a number of reducible backgrounds are expected to limit 


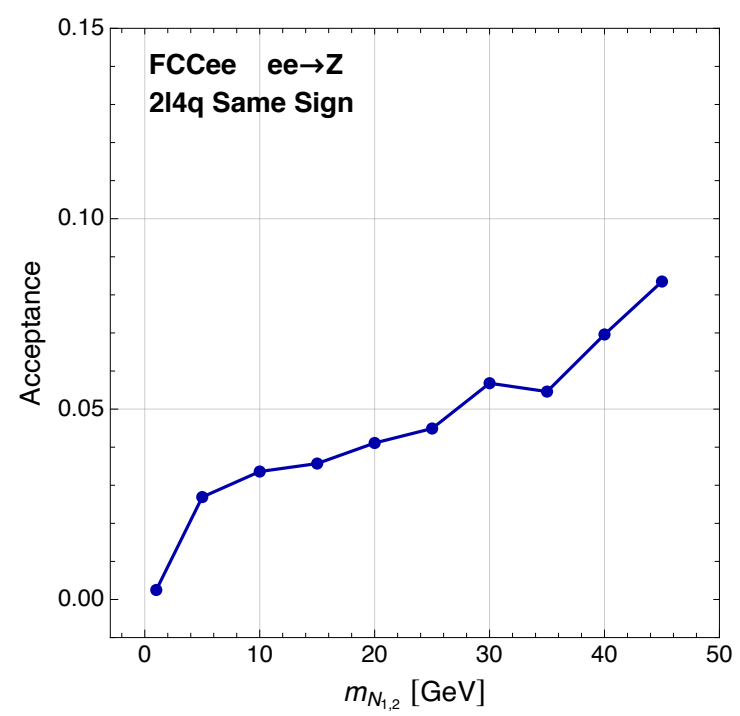

Figure 11. Parton level acceptances for the $2 \ell 4 q$ final state in the $Z$ channel topology with a leptonically decaying $Z$ boson and with the selection cut described in the main text.

the statistical reach of the $Z$ boson sample, depending both on the limited detector performances and on the collider characteristics. We then expect that a realistic background analysis for the $Z \rightarrow \ell^{+} \ell^{+} 4 q$ channel might degrade the ideal background free estimate in a non negligible way.

As an example of how different reducible backgrounds can degrade the $Z$ sample sensitivity, we will consider below the possible background arising from the limited leptoncharge identification power of a LHC-like detector. Indeed, conservation of lepton number implies that no genuine irreducible $Z \rightarrow \ell^{+} \ell^{+} 4 q$ background arises in the SM. As regarding the $Z \rightarrow \ell^{+} \ell^{+} 2 \nu 4 q$ background, for which we expect the cross-section to be relatively small, this might also be efficiently reduced by asking for limited missing energy in the events. On the other hand, a realistic analysis of the reducible SM background $Z \rightarrow \ell^{+} \ell^{-} 4 q$, where a lepton charge is misidentified, or an hadron is misidentified as a lepton, would require a dedicated full simulation at the experimental level, which is beyond the scope of the current work. For the lepton-charge misidentification effect, assuming LHC performances of lepton charge identification, we provide an approximate estimate of the corresponding background by applying a (flat) mis-identification probability factor of $\epsilon_{\text {misID }}^{\ell}=10^{-3}$ [63] to the partonic cross-section $e^{+} e^{-} 4 q$ and $\mu^{+} \mu^{-} 4 q$ after the signal selections described in the text. ${ }^{10}$ With this procedure we obtain a background yield of $\sigma_{\ell^{+} \ell^{-} 4 q} \times 2 \times \epsilon_{\mathrm{misID}}^{\ell}\left(1-\epsilon_{\mathrm{misID}}^{\ell}\right) \simeq 130 \mathrm{fb} \times \epsilon_{\mathrm{misID}}^{\ell}\left(1-\epsilon_{\mathrm{misID}}^{\ell}\right) \simeq 0.26 \mathrm{fb}$, which we use as estimate for our analysis.

We show our results for $\mathbf{B P} \mathbf{1}_{\mathbf{N H}}$ in figure 12 in the $m_{N}-\mathrm{BR}\left(Z \rightarrow N_{1} N 2\right)$ plane. As for the $\mathcal{O}_{N H}$ case, in computing our limits we are summing on all the lepton flavor combinations from the $N_{1} N_{2}$ decay and we are considering all lepton charges configurations

\footnotetext{
${ }^{10}$ Note that at the LHC the probability of mis-identifying the charge of a muon is generally negligible, since muon tracks are measured both in the inner detector and in the muon spectrometer.
} 

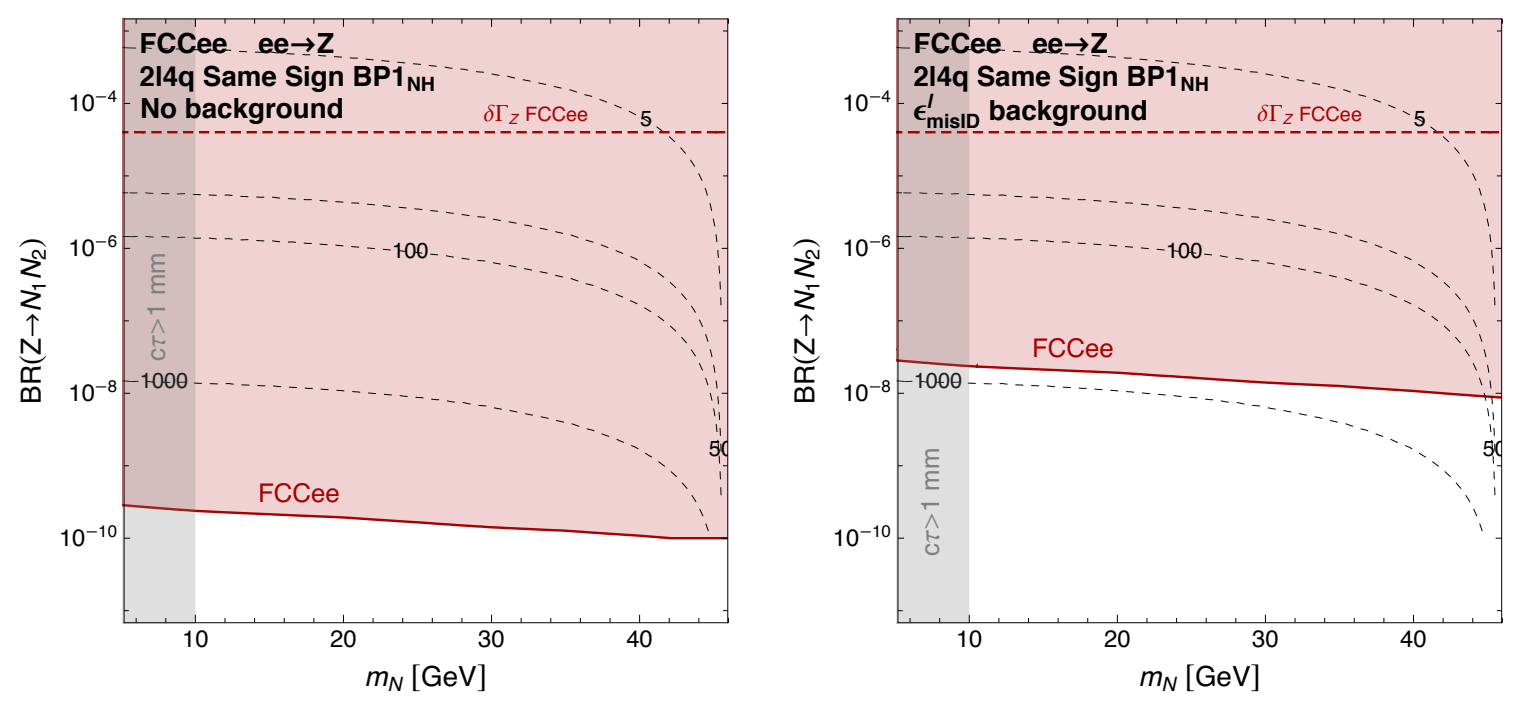

Figure 12. $95 \%$ CL exclusion for $\mathbf{B P} \mathbf{1}_{\mathbf{N H}}$ from prompt searches in the s-channel $Z$ production channel. The red shaded area represent the exclusion for the case of FCC-ee. Also shown as a red dashed line the limits arising from the measurement of the $Z$ boson total width and as gray dashed lines the isocontours of NP scale $\Lambda$ in $\mathrm{TeV}$. In the gray shaded area $c \tau>1 \mathrm{~mm}$ and the $\mathrm{RH}$ neutrino cannot decay promptly without being excluded by experimental searches. In the left plot we assume zero background while in the right plot we include the SM reducible background arising from lepton charge mis-identification.

that give rise to a SS lepton final state. In the left panel we assume zero background, while in the right panel we estimate the background coming from lepton charge mis-identification following the procedure outline above. The red region is the one that will be probed by the FCC-ee, while the horizontal dashed red line represent the expected sensitivity on the $Z$ boson width of $100 \mathrm{keV}$ [34]. From the figure it is clear that, when considering the presence of the lepton charge mis-identification background, FCC-ee will be able to exclude values of $\operatorname{BR}\left(Z \rightarrow N_{1} N_{2}\right)$ down to roughly $10^{-8}$. This corresponds to a reach on $\Lambda \simeq 10^{3} \mathrm{TeV}$. Given that this operator can only be generated at loop-level, this bounds is rescaled by a factor $16 \pi^{2}$ when mapped into the physical masses and couplings of an ultraviolet complete model. We stress that the present estimate might be further degraded by other background sources, such as other particle identification inefficiencies which will be strongly dependent on the actual detector performance.

\subsection{Displaced decay}

For the case in which the $\mathrm{RH}$ neutrinos decay displaced from the primary vertex we follow again the strategy outlined in section 4.2. As opposed to the case of prompt decay, in this case we expect negligible irreducible SM background. We show in the left panel of figure 13 the FCC-ee reach for the $\mathbf{B P} \mathbf{1}_{\mathbf{I H}}$ benchmark, projected in the $m_{N}-\left|\theta_{e}\right|^{2}$. We fix $\operatorname{BR}\left(Z \rightarrow N_{1} N_{2}\right)=10^{-10}$ and assume different efficiencies $\epsilon_{\text {displ }}$, as reported in the plots. The gray shaded are represents the see-saw limit, while the values of the mixing angles above the gray dashed line are excluded by experimental searches. As for the case of the 

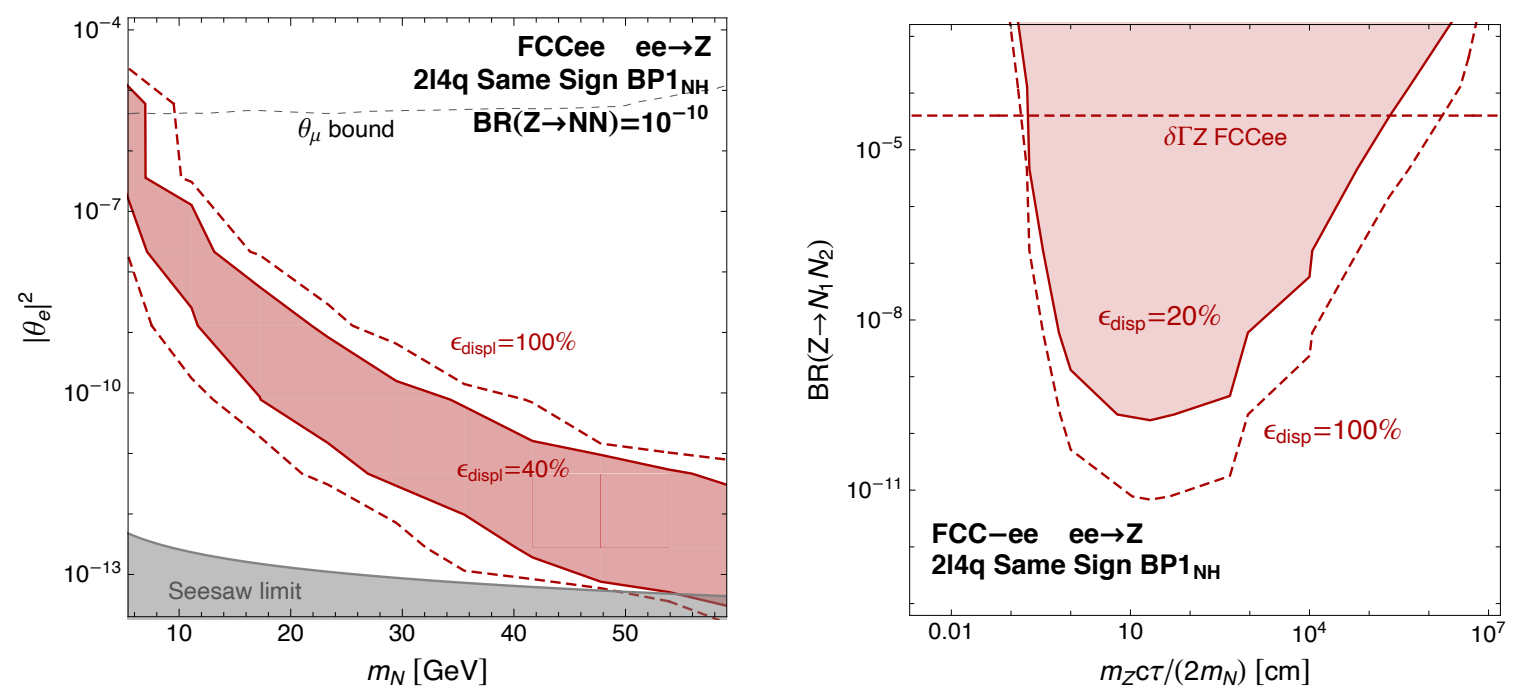

Figure 13. Left: $95 \%$ CL exclusion for $\mathbf{B P} 1_{\mathbf{N H}}$ in the $m_{N}-\left|\theta_{e}\right|^{2}$ plane from displaced decay searches assuming $\operatorname{BR}\left(Z \rightarrow N_{1} N_{2}\right)=10^{-10}$ for the case of FCC-ee. The gray dashed line represents the limit on the mixing angle arising from existing experimental searches. In the gray shaded region the lightness of the neutrino masses cannot be explained by the see-saw mechanism. Right: $95 \%$ CL exclusion for $\mathbf{B P} \mathbf{1}_{\mathbf{N H}}$ in the $\frac{m_{Z}}{2 m_{N}} c \tau-\mathrm{BR}\left(h \rightarrow N_{1} N_{2}\right)$ plane from displaced decay searches. The horizontal red dashed line represents the limit arising from the FCC-ee $Z$ boson total width measurement with an uncertainty of $100 \mathrm{keV}$.

$\mathcal{O}_{N H}$ operator our results show that the search for $\mathrm{RH}$ neutrinos arising from $Z$ decay and decaying displaced from the primary vertex can offer a great handle in testing the active-sterile mixing angle. Again this is due to the fact that the production cross-section does not depend on this quantity, but only on the $Z$ decay rate into the NP final state. In the right panel of the same figure we show instead the FCC-ee reach projected in the $\frac{m_{Z}}{2 m_{N}} c \tau-\operatorname{BR}\left(h \rightarrow N_{1} N_{2}\right)$ plane. We do this again for two different choices of $\epsilon_{\text {displ }}$ as reported in the plot. All together we see that FCC-ee will be able to test values of the $Z$ boson exotic branching ratio down to $10^{-9}$ for $\epsilon_{\text {disp. }}=20 \%$, largely surpassing the limit arising from the FCC-ee $Z$ boson total width measurement with an uncertainty of $100 \mathrm{keV}$, which is represented by the horizontal red dashed line.

\subsection{Detector stable}

The last case we study is again the possibility that the $\mathrm{RH}$ neutrinos lifetime is large enough to cause them to decay outside the detector. Similarly to the $\mathcal{O}_{N H}$ operator, in this case the decay will contribute to the invisible $Z$ width. FCC-ee will measure the ratio $R_{\nu}=\Gamma_{Z \rightarrow \text { inv }} / \Gamma_{Z \rightarrow \ell \ell}$ at the level of $0.27 \times 10^{-3}$ [52] which, under the SM hypothesis, correspond to an additional contribution to invisible decay width of the $Z$ boson smaller than $\sim 135 \mathrm{KeV}$. This limit can be translated in a bound on the NP scale $\Lambda$ which will be constrained by this measurement to be $\Lambda \gtrsim 16 \mathrm{TeV}$ for $m_{N} \sim 10 \mathrm{GeV}$ while this limits degrade down to $\Lambda \gtrsim 9 \mathrm{TeV}$ for $m_{N}=35 \mathrm{GeV}$ due to phase space effect. Above this mass threshold their decay will happen inside the detector, see figure 2 . 


\section{The impact of $d=6$ operators}

We have so far considered the phenomenology induced by the presence of $d=5$ operators in the $\nu$ SMEFT, discussing how they can induce additional $\mathrm{RH}$ neutrinos production and decay modes and showing how they can be efficiently tested at future Higgs Factories. It is important however to notice that at $d=6$ many more operators are present, and they might give observable signatures. For example, among the operators involving the Higgs field, of particular interest are

$$
\begin{aligned}
\mathcal{O}_{L N B} & =\alpha_{L N B}\left(\bar{L} \sigma^{\mu \nu} N\right) B_{\mu \nu} \tilde{H}, \\
\mathcal{O}_{L N W} & =\alpha_{L N W}\left(\bar{L} \sigma^{\mu \nu} N\right) W_{\mu \nu} \tilde{H} .
\end{aligned}
$$

They will trigger the decay to an active neutrino and a photon with a rate

$$
\Gamma\left(N \rightarrow \nu_{i} \gamma\right) \simeq \frac{m_{N}^{3} v^{2}}{4 \pi \Lambda^{4}}\left(\alpha_{L N B}+s_{w} \alpha_{L N W}\right)^{2},
$$

where, with an abuse of notation, we also indicate with $\alpha_{L N B}$ and $\alpha_{L N W}$ the relevant entries of the corresponding Wilson coefficient matrices. Also this decay mode can in principle dominate over the one induced via the active-sterile mixing in some regions of the parameter space. A detailed analysis would proceed in a very similar fashion to the one described in section 5 for the dipole operator $\mathcal{O}_{N B}$, with however an additional suppression due to their higher dimensionality. These two operators have also been recently studied in the context of LHC in ref. [31] where a bound on $\Lambda \gtrsim 2.2 \mathrm{TeV}$ has been obtained via the $p p \rightarrow h \rightarrow \nu N \gamma$ with subsequent decay $N \rightarrow \nu \gamma$. Notice that in deriving this bound the Authors of [31] have considered also the Higgs decay to be triggered by the $d=6$ operators of eq. (6.1).

At $d=6$ it is also interesting to notice the presence of four fermions operators. In the case of future Higgs Factories of particular relevance is

$$
\mathcal{O}_{N e}=\alpha_{N E}\left(\bar{N} \gamma^{\mu} N\right)\left(\bar{e}_{R} \gamma_{\mu} e_{R}\right),
$$

which triggers a direct production channel for the $\mathrm{RH}$ neutrinos pair with a rate

$$
\sigma(e e \rightarrow N N) \simeq\left(\frac{\sqrt{s}}{8 \pi \Lambda^{2}} \alpha_{N E}\right)^{2} \beta\left(1+\frac{\beta^{3}}{3}\right),
$$

where $\beta=\sqrt{1-\frac{4 m_{N}^{2}}{s}}$, which gives

$$
\sigma(e e \rightarrow N N) \simeq 1 \mathrm{fb}\left(\frac{2.5 \mathrm{TeV}}{\Lambda}\right)^{4}\left(\frac{\sqrt{s}}{240 \mathrm{GeV}}\right)^{2}
$$

for $m_{N}=10 \mathrm{GeV}$. However, unless the coefficients of the $d=5$ operator $\mathcal{O}_{N H}$ and the one of the $d=6$ operator $\mathcal{O}_{N e}$ have a different scaling, for equal NP scale $\Lambda$ the production induced by the former is always dominant with respect to the latter for regions where the additional Higgs decay width is compatible with current constraints, $\Lambda \gtrsim 50 \mathrm{TeV}$. 
The situation can be drastically different in the case where the underlying theory has a particular symmetry, as in the case of Minimal Flavor violation recently analyzed in [51]. In this case the Higgs operator receives an extra suppression of a factor $\sim m_{N} / \Lambda$, while four fermions operators as $\mathcal{O}_{N e}$ will not be affected by the insertion of any spurion. In this case the main production mechanism, especially for low neutrino masses and large center of mass energies, will be via the one through the $d=6$ operators.

\section{Conclusions}

The observed pattern of neutrino masses and oscillation parameters require extending the Standard Model. One of the simplest possibilities is to add to the SM particle content two or more $\mathrm{RH}$ neutrinos. In this framework active neutrino masses compatible with current experimental measurements are generated via the see-saw mechanism, through an interplay of the active-sterile Yukawa coupling and the RH neutrinos Majorana mass. Naturalness consideration and the observation of a large baryon asymmetry in the Universe, motivates the study of scenarios where RH neutrinos have a mass $M_{N}$ at around the EW scale $v$, hence testable at current and future collider experiments. In general, such $\mathrm{RH}$ neutrino states are assumed to be produced through mixing with the SM sector, which is also responsible for their decay. The presence of additional New Physics at a scale $\Lambda \gg v, M_{N}$ can drastically modify their phenomenology and have thus a huge impact for present and future experimental search strategies. The impact of these extra deformations can be parametrized at low energy as an effective field theory with $d>4$ operators built out from SM and $\mathrm{RH}$ neutrino fields. In this work we have focused on the effect of the two new $d=5$ operators containing both the SM and the RH neutrinos, $\mathcal{O}_{N H}$ and $\mathcal{O}_{N B}$. They induce additional production modes for $\mathrm{RH}$ neutrino pairs through the decay of the Higgs and the $Z$ boson respectively. We have then studied the phenomenology of these two operators at future Higgs Factories, such as FCC-ee, CEPC, ILC and CLIC-380, for different regimes of $\mathrm{RH}$ neutrinos lifetimes. In particular we have considered $\mathrm{RH}$ neutrinos with prompt, displaced or outside the detector decays. For both operators we have shown that, in favorable scenarios for detector performance and systematics, future Higgs Factories have a great potential in testing the NP scale $\Lambda$, well above the limit that can be set by indirect probes such as the search for additional untagged Higgs decay or the measurement of the $Z$ boson decay width. In particular, in the case of prompt decays the Higgs and $Z$ branching ratios into $N N$ pairs can be tested at the level of $10^{-3}$ and $10^{-8}$, respectively, in the most favorable scenarios, while search for displaced decays can probe active-sterile mixing angles down to the see-saw limit of $\left|\theta_{e}\right|^{2} \sim 10^{-13}$. We have moreover discussed the possibility of disentangling the underlying flavor structure should a signal be observed in future experiments, and commented on possible additional signatures that can be triggered by $d=6$ operators, and that deserve a separate and dedicated study. 


\section{Acknowledgments}

We thank Roberto Franceschini for comments on the draft. EB acknowledges financial support from FAPESP under contracts 2015/25884-4 and 2019/15149-6 and is indebted to the Theoretical Particle Physics and Cosmology group at King's College London for its hospitality. AC acknowledges hospitality from the MPP of Munich. AC acknowledges support from the Generalitat Valenciana (GVA) through the GenT program (CIDEGENT/2018/019). $\mathrm{PH}$ acknowledge support from the GVA project PROMETEO/2019/083, as well as the national grant FPA2017-85985-P and the European grant H2020-MSCA-ITN-2019//860881HIDDeN.

Open Access. This article is distributed under the terms of the Creative Commons Attribution License (CC-BY 4.0), which permits any use, distribution and reproduction in any medium, provided the original author(s) and source are credited.

\section{References}

[1] P. Minkowski, $\mu \rightarrow$ er at a Rate of One Out of $10^{9}$ Muon Decays?, Phys. Lett. B 67 (1977) 421 [INSPIRE].

[2] R.N. Mohapatra and G. Senjanović, Neutrino Mass and Spontaneous Parity Nonconservation, Phys. Rev. Lett. 44 (1980) 912 [INSPIRE].

[3] T. Yanagida, Horizontal gauge symmetry and masses of neutrinos, Conf. Proc. C 7902131 (1979) 95 [INSPIRE].

[4] M. Gell-Mann, P. Ramond and R. Slansky, Complex Spinors and Unified Theories, Conf. Proc. C $\mathbf{7 9 0 9 2 7}$ (1979) 315 [arXiv: 1306.4669] [inSPIRE].

[5] E.K. Akhmedov, V.A. Rubakov and A.Y. Smirnov, Baryogenesis via neutrino oscillations, Phys. Rev. Lett. 81 (1998) 1359 [hep-ph/9803255] [INSPIRE].

[6] T. Asaka and M. Shaposhnikov, The $\nu M S M$, dark matter and baryon asymmetry of the universe, Phys. Lett. B 620 (2005) 17 [hep-ph/0505013] [INSPIRE].

[7] W.-Y. Keung and G. Senjanović, Majorana Neutrinos and the Production of the Right-handed Charged Gauge Boson, Phys. Rev. Lett. 50 (1983) 1427 [InSPIRE].

[8] A. Ferrari et al., Sensitivity study for new gauge bosons and right-handed Majorana neutrinos in pp collisions at $s=14 \mathrm{TeV}$, Phys. Rev. D 62 (2000) 013001 [INSPIRE].

[9] M.L. Graesser, Experimental Constraints on Higgs Boson Decays to TeV-scale Right-Handed Neutrinos, arXiv:0705.2190 [INSPIRE].

[10] F. del Aguila and J.A. Aguilar-Saavedra, Distinguishing seesaw models at LHC with multi-lepton signals, Nucl. Phys. B 813 (2009) 22 [arXiv:0808.2468] [INSPIRE].

[11] P.S. Bhupal Dev, R. Franceschini and R.N. Mohapatra, Bounds on TeV Seesaw Models from LHC Higgs Data, Phys. Rev. D 86 (2012) 093010 [arXiv:1207.2756] [InSPIRE].

[12] J.C. Helo, M. Hirsch and S. Kovalenko, Heavy neutrino searches at the LHC with displaced vertices, Phys. Rev. D 89 (2014) 073005 [Erratum ibid. 93 (2016) 099902] [arXiv:1312.2900] [INSPIRE]. 
[13] FCC-Ee study Team collaboration, Search for Heavy Right Handed Neutrinos at the FCC-ee, Nucl. Part. Phys. Proc. 273-275 (2016) 1883 [arXiv:1411.5230] [InSPIRE].

[14] A. Abada, V. De Romeri, S. Monteil, J. Orloff and A.M. Teixeira, Indirect searches for sterile neutrinos at a high-luminosity Z-factory, JHEP 04 (2015) 051 [arXiv:1412.6322] [INSPIRE].

[15] Y. Cui and B. Shuve, Probing Baryogenesis with Displaced Vertices at the LHC, JHEP 02 (2015) 049 [arXiv: 1409.6729] [InSPIRE].

[16] S. Antusch and O. Fischer, Testing sterile neutrino extensions of the Standard Model at future lepton colliders, JHEP 05 (2015) 053 [arXiv: 1502.05915] [INSPIRE].

[17] A.M. Gago, P. Hernández, J. Jones-Pérez, M. Losada and A. Moreno Briceño, Probing the Type I Seesaw Mechanism with Displaced Vertices at the LHC, Eur. Phys. J. C 75 (2015) 470 [arXiv:1505.05880] [INSPIRE].

[18] S. Antusch, E. Cazzato and O. Fischer, Displaced vertex searches for sterile neutrinos at future lepton colliders, JHEP 12 (2016) 007 [arXiv:1604.02420] [INSPIRE].

[19] A. Caputo, P. Hernández, M. Kekic, J. López-Pavón and J. Salvado, The seesaw path to leptonic CP-violation, Eur. Phys. J. C 77 (2017) 258 [arXiv:1611.05000] [INSPIRE].

[20] A. Das, P.S.B. Dev and C.S. Kim, Constraining Sterile Neutrinos from Precision Higgs Data, Phys. Rev. D 95 (2017) 115013 [arXiv:1704.00880] [InSPIRE].

[21] A. Caputo, P. Hernández, J. Lopez-Pavon and J. Salvado, The seesaw portal in testable models of neutrino masses, JHEP 06 (2017) 112 [arXiv: 1704.08721] [INSPIRE].

[22] A. Abada, N. Bernal, M. Losada and X. Marcano, Inclusive Displaced Vertex Searches for Heavy Neutral Leptons at the LHC, JHEP 01 (2019) 093 [arXiv:1807.10024] [INSPIRE].

[23] P. Hernández, J. Jones-Pérez and O. Suarez-Navarro, Majorana vs Pseudo-Dirac Neutrinos at the ILC, Eur. Phys. J. C 79 (2019) 220 [arXiv:1810.07210] [INSPIRE].

[24] J. Jones-Pérez, J. Masias and J.D. Ruiz-Álvarez, Search for Long-Lived Heavy Neutrinos at the LHC with a VBF Trigger, Eur. Phys. J. C 80 (2020) 642 [arXiv:1912.08206] [INSPIRE].

[25] J. Kersten and A.Y. Smirnov, Right-Handed Neutrinos at CERN LHC and the Mechanism of Neutrino Mass Generation, Phys. Rev. D 76 (2007) 073005 [arXiv:0705.3221] [INSPIRE].

[26] M.B. Gavela, T. Hambye, D. Hernandez and P. Hernández, Minimal Flavour Seesaw Models, JHEP 09 (2009) 038 [arXiv:0906.1461] [INSPIRE].

[27] M.L. Graesser, Broadening the Higgs boson with right-handed neutrinos and a higher dimension operator at the electroweak scale, Phys. Rev. D 76 (2007) 075006 [arXiv: 0704.0438] [INSPIRE].

[28] A. Aparici, K. Kim, A. Santamaria and J. Wudka, Right-handed neutrino magnetic moments, Phys. Rev. D 80 (2009) 013010 [arXiv:0904.3244] [INSPIRE].

[29] F. del Aguila, S. Bar-Shalom, A. Soni and J. Wudka, Heavy Majorana Neutrinos in the Effective Lagrangian Description: Application to Hadron Colliders, Phys. Lett. B 670 (2009) 399 [arXiv:0806.0876] [INSPIRE].

[30] Y. Liao and X.-D. Ma, Operators up to Dimension Seven in Standard Model Effective Field Theory Extended with Sterile Neutrinos, Phys. Rev. D 96 (2017) 015012 [arXiv: 1612.04527] [INSPIRE]. 
[31] J.M. Butterworth, M. Chala, C. Englert, M. Spannowsky and A. Titov, Higgs phenomenology as a probe of sterile neutrinos, Phys. Rev. D 100 (2019) 115019 [arXiv: 1909.04665] [INSPIRE].

[32] J. Alcaide, S. Banerjee, M. Chala and A. Titov, Probes of the Standard Model effective field theory extended with a right-handed neutrino, JHEP 08 (2019) 031 [arXiv:1905.11375] [INSPIRE].

[33] J. de Vries, H.K. Dreiner, J.Y. Günther, Z.S. Wang and G. Zhou, Long-lived Sterile Neutrinos at the LHC in Effective Field Theory, arXiv:2010.07305 [INSPIRE].

[34] TLeP Design Study Working Group collaboration, First Look at the Physics Case of TLEP, JHEP 01 (2014) 164 [arXiv:1308.6176] [INSPIRE].

[35] FCC collaboration, FCC-ee: The Lepton Collider: Future Circular Collider Conceptual Design Report Volume 2, Eur. Phys. J. ST 228 (2019) 261 [inSPIRE].

[36] FCC collaboration, FCC Physics Opportunities: Future Circular Collider Conceptual Design Report Volume 1, Eur. Phys. J. C 79 (2019) 474 [inSPIRE].

[37] N. Alipour Tehrani et al., FCC-ee: Your Questions Answered, in CERN Council Open Symposium on the Update of European Strategy for Particle Physics, A. Blondel and P. Janot eds. (2019) [arXiv: 1906. 02693] [INSPIRE].

[38] CEPC Study Group collaboration, CEPC Conceptual Design Report: Volume 1 Accelerator, arXiv:1809.00285 [INSPIRE].

[39] CEPC Study Group collaboration, CEPC Conceptual Design Report: Volume 2 Physics $\mathcal{E}$ Detector, arXiv:1811.10545 [INSPIRE].

[40] T. Behnke et al. eds., The International Linear Collider Technical Design Report - Volume 1: Executive Summary, arXiv:1306.6327 [INSPIRE].

[41] H. Baer et al., eds., The International Linear Collider Technical Design Report - Volume 2: Physics, arXiv:1306.6352 [INSPIRE].

[42] P. Bambade et al., The International Linear Collider: A Global Project, arXiv:1903.01629 [INSPIRE].

[43] R. Franceschini et al., The CLIC Potential for New Physics, arXiv:1812.02093 [InSPIRE].

[44] CLIC and CLICDP collaborations, P. Roloff, R. Franceschini, U. Schnoor and A. Wulzer, eds., The Compact Linear $e^{+} e^{-}$Collider (CLIC): Physics Potential, arXiv:1812.07986 [INSPIRE].

[45] S. Weinberg, Baryon and Lepton Nonconserving Processes, Phys. Rev. Lett. 43 (1979) 1566 [INSPIRE].

[46] B. Pontecorvo, Inverse beta processes and nonconservation of lepton charge, Sov. Phys. JETP 7 (1958) 172 [INSPIRE].

[47] Z. Maki, M. Nakagawa and S. Sakata, Remarks on the unified model of elementary particles, Prog. Theor. Phys. 28 (1962) 870 [InSPIRE].

[48] J.A. Casas and A. Ibarra, Oscillating neutrinos and $\mu \rightarrow e, \gamma$, Nucl. Phys. B 618 (2001) 171 [hep-ph/0103065] [INSPIRE].

[49] K. Bondarenko, A. Boyarsky, D. Gorbunov and O. Ruchayskiy, Phenomenology of GeV-scale Heavy Neutral Leptons, JHEP 11 (2018) 032 [arXiv:1805.08567] [INSPIRE]. 
[50] P. Hernández, M. Kekic, J. López-Pavón, J. Racker and J. Salvado, Testable Baryogenesis in Seesaw Models, JHEP 08 (2016) 157 [arXiv:1606.06719] [INSPIRE].

[51] D. Barducci, E. Bertuzzo, A. Caputo and P. Hernández, Minimal flavor violation in the see-saw portal, JHEP 06 (2020) 185 [arXiv: 2003.08391] [INSPIRE].

[52] J. de Blas et al., Higgs Boson Studies at Future Particle Colliders, JHEP 01 (2020) 139 [arXiv: 1905.03764] [INSPIRE].

[53] ALEPH, DELPHI, L3, OPAL AND SLD collaborations, LEP Electroweak Working Group, SLD Electroweak Group, SLD Heavy Flavour Group, Precision electroweak measurements on the $Z$ resonance, Phys. Rept. 427 (2006) 257 [hep-ex/0509008] [INSPIRE].

[54] J. Alwall et al., The automated computation of tree-level and next-to-leading order differential cross sections, and their matching to parton shower simulations, JHEP 07 (2014) 079 [arXiv: 1405.0301] [INSPIRE].

[55] E. Conte, B. Fuks and G. Serret, MadAnalysis 5, A User-Friendly Framework for Collider Phenomenology, Comput. Phys. Commun. 184 (2013) 222 [arXiv:1206.1599] [InSPIRE].

[56] E. Conte, B. Dumont, B. Fuks and C. Wymant, Designing and recasting LHC analyses with MadAnalysis 5, Eur. Phys. J. C $\mathbf{7 4}$ (2014) 3103 [arXiv: 1405.3982] [InSPIRE].

[57] B. Dumont et al., Toward a public analysis database for LHC new physics searches using MADANALYSIS 5, Eur. Phys. J. C 75 (2015) 56 [arXiv:1407.3278] [INSPIRE].

[58] Belle collaboration, Search for heavy neutrinos at Belle, Phys. Rev. D 87 (2013) 071102 [Erratum ibid. 95 (2017) 099903] [arXiv: 1301.1105] [INSPIRE].

[59] LHCb collaboration, Search for massive long-lived particles decaying semileptonically in the LHCb detector, Eur. Phys. J. C 77 (2017) 224 [arXiv:1612.00945] [InSPIRE].

[60] DELPHI collaboration, Search for neutral heavy leptons produced in $Z$ decays, Z. Phys. C 74 (1997) 57 [Erratum ibid. 75 (1997) 580] [INSPIRE].

[61] Particle Data Group collaboration, Review of Particle Physics, PTEP 2020 (2020) 083C01 [INSPIRE].

[62] T.H. Tran, V. Balagura, V. Boudry, J.-C. Brient and H. Videau, Reconstruction and classification of tau lepton decays with ILD, Eur. Phys. J. C 76 (2016) 468 [arXiv: 1510.05224] [INSPIRE].

[63] ATLAS collaboration, Electron reconstruction and identification in the ATLAS experiment using the 2015 and 2016 LHC proton-proton collision data at $\sqrt{s}=13 \mathrm{TeV}$, Eur. Phys. J. C 79 (2019) 639 [arXiv:1902.04655] [INSPIRE]. 